\title{
UN NUEVO PERFIL DEL PROTECCIONISMO ESPAÑOL DURANTE LA RESTAURACIÓN, $1875-1930$ *
}

\author{
ANTONIO TENA JUNGUITO
}

Universidad Carlos III de Madrid

\section{RESUMEN}

Este artículo discute una serie de nuevos indicadores alternativos para medir la protección nominal en España entre 1875 a 1930. Los nuevos indicadores apuntan la existencia de un importante sesgo a la baja del índice de la protección nominal convencional, tanto en los años de mayor incremento como en sus sectores protagonistas. Confirman, por un lado, que los niveles de protección en España en los inicios de la Restauración eran ya relativamente altos, por el otro, niegan una tendencia plana y proponen una alcista aunque cíclica en su evolución con su punto de mayor auge en los años veinte. El sesgo industrial es permanente, sin embargo la industria sale ganando en mayor medida con los aranceles que acentúan claramente la protección y la agricultura con los que la relajan o moderan. El alto punto de partida, la tendencia cíclica alcista y el permanente sesgo industrial apoyan la hipótesis tradicional de que existió una fuerte influencia del sector industrial en el diseño de la política comercial española y contradicen la hipótesis del carácter «forzado» o «fortuito» del viraje proteccionista de 1891.

* Partes de este trabajo han sido presentadas en versiones distintas en diversos seminarios: Universidad Internacional Menéndez y Pelayo, V Congreso de la Asociación de Historia Económica, Eleventh International Economic History Asociation Congress, Universidad de Valencia, Universidad Central de Barcelona y Universidad Carlos III. Debo agradecer las críticas y los comentarios, entre otros, de Concha Beltrán, Giovanni Federico, Pedro Fraile, Agustín Llona, Elena Martínez, Jordi Palafox, Leandro Prados de la Escosura, Pablo Sánchez León y Daniel Tirado, así como por los útiles y detallados comentarios de un evaluador anónimo. Tampoco puedo dejar de dar las gracias por su colaboración en la elaboración de la base de datos a Laura Cervero, Raimundo Fernández Cuesta y Cristina Cambeiro. Este proyecto ha sido financiado con una beca del Ministerio de Educación CGYCIT: PB 94/073. 


\section{ABSTRACT}

This article discusses a new series of alternative indexes to measure Spanish protectionism in the period 1875-1930. The new indexes show that the biases downwards of the conventional index are relevant specially for the protection increase at given years and sectors. They confirm the relatively high level of protection at the beginning of the «Restauracion» in the 1870's but contradict the stable level of protection before First World War and put forward an upward cyclical trend until the 1920's. From the beginning of the period Spanish protection was mainly industrial. When the general protection index is moderate, agriculture improves its position in relation to industry, and when protection increases the reverse occurs. The high level of departure, the upward cyclical trend and the permanent industrial bias support the traditional hypothesis of the existence of a strong influence of the industrial sector in Spanish comercial policy design and deny the «compulsory» or «accidental» explanation of the Spanish return to protection in the 1890's.

\section{INTRODUCCIÓN}

Aunque pocos lo hagan explícito la mayoría de los historiadores económicos españoles parece dar por sentado la importancia del tema de la protección para el crecimiento económico español en el último cuarto del siglo Xxx y primer tercio del $\mathrm{xx}$. En términos generales, se puede decir que una gran parte de los estudiosos es partidaria de una valoración positiva de la incidencia de la protección sobre el crecimiento económico. Aunque esta apreciación positiva se expresa con matices muy diversos. Por un lado, la mayoría son críticos, en coincidencia con Flores de Lemus, con los proteccionismos superpuestos que implicaría la propuesta retórica de la «protección integral». Pero, por el otro, los mismos también sugieren que una opción alternativa más librecambista era demasiado arriesgada. En su opinión, el atraso de la economía española implicaba serias dificultades para iniciar procesos de especialización, y en consecuencia, tanto los factores como los recursos podrían desaparecer, en vez de trasladarse hacia otras actividades productivas con ventaja comparativa, si se les obligaba a hacer frente a la competencia internacional ${ }^{~}$. Es decir, la mayoría de los autores se refugian en lo «inevitable» de la opción proteccionista, bien por razones

1 Como apunta Raymond Carr: «La exigencia de proteccionismo era sólo un aspecto del pesimismo económico que siguió al fin del auge de la restauración», Carr (1990), p. 381. Este pesimismo es el mismo que comparte la literatura ai negar que existieran alternativas a la protección. 
políticas $^{2}$ o económicas ${ }^{3}$. O lo que es lo mismo, en la creencia de que una alternativa menos proteccionista hubiera sido políticamente inviable y globalmente negativa en términos económicos ${ }^{4}$. Sólo en los últimos años algunos autores han resaltado la posibilidad de que en ausencia de una protección tan alta la economía española habría reaccionado de forma similar a como lo hicieron otros países en estos mismos años, adelantando parte de las transformaciones que finalmente se llevaron a cabo en la segunda mitad del siglo $\mathrm{xx}^{5}$.

Realmente se puede decir que el tema del proteccionismo fue una parte central del debate político durante la segunda mitad del siglo XIX y naturalmente ha tenido su continuación en el debate historiográfico. El estudio de los procesos de elaboración, los instrumentos empleados y los

2 «Era inconcebible que los políticos se arriesgaran a las consecuencias políticas y sociales de sacrificar la agricultura castellana y la industria vasca», Carr (1990), p. 381.

${ }_{3}^{3}$ Quizá la posición proteccionista de Flores de Lemus sea una de las más conscientes de los costes que implicaba. En este sentido puso muchas veces de manifiesto que la protección conseguida por la industria no era gratuita y era pagada por los exportadores agrarios. Se ha resaltado muchas veces su preocupación por la posición retórica del «proteccionismo integral» debido al hecho de que esto suponía protecciones superpuestas que dañaban inútilmente a la agricultura exportadora sin incrementar la protección efectiva de la industria. Pero en definitiva compartía la posición pesimista de que la industria desaparecería sin protección y era, por tanto, partidario de la misma: «Y bien, suprimid esa industria agrícola de exportación y sus congéneres y se hunde la constitución industrial de vizcaínos y catalanes. Pero suprimir Vasconia y Cataluña y veréis qué pasa con la agricultura española.» Para continuar: «(...) hecha esta combinación de industria con agricultura exportadora, cada uno de los agricultores exportadores que pagan el proteccionismo tenga como es natural, menos que si ese proteccionismo no existiera; pero todos ellos juntos tengan más que si no existiera la industria. Y todos ellos juntos con la Nación y la Patria», Flores de Lemus (1928), p. 42.

4 Vicens Vives tiende a mostrar una imagen más bien positiva de la vuelta al proteccionismo presentando quizás el reforzamiento de la misma como algo inevitable. En relación al arancel de 1891 dice: «Bajo ese régimen de proteccionismo se desarrolló la industria siderúrgica española y alcanzó su mayor auge la textil (...) la pérdida de las colonias obligó a España a defenderse, y nada tiene de extraño que el tres de marzo de 1906 se diera a España un arancel decididamente proteccionista», Vicens (1990), p. 645. Siguiendo la línea de Vicens, Josep Fontana también mantiene una opinión firme sobre la inevitabilidad de la protección: «El triunfo del proteccionismo arancelario es el resultado inevitable de la situación objetiva de la economía española», Fontana (1973), p. 185. Esta misma opinión, aunque más matizada, la comparte Jordi Maluquer (1987): «Quizá el problema principal no estuviera en la protección misma, sino en su reforzamiento cuando hubiera resultado más positiva su paulatina reducción», Maluquer (1987), p. 71. Más adelante confirma la imposibilidad de llevar a cabo esta reducción en el arancel de 1906: «La trayectoria seguida por la economia española en 1898-1921, y la política económica que la instrumentó, respondían a unos condicionamientos casi ineludibles. Probablemente no había alternativa». Aunque termina reconociendo que «probablemente los costes de la opción nacionalista fueron demasiado elevados» (p. 99).

5 Ver Tortella (1981, 1994), Prados (1982, 1988), Carreras (1984), Fraile (1985, 1991), Tena (1992a, 1995). 
objetivos que perseguían las leyes arancelarias ha concentrado los mayores esfuerzos investigadores de estos últimos años ${ }^{6}$. Sin embargo, aunque se ha profundizado y enriquecido la visión del período este tipo de estudio ha desenfocado algunas de las preguntas esenciales sobre las que articular los términos del debate sobre el papel de la política comercial en el crecimiento económico español del último cuarto del siglo XIX y primer tercio del xx. Parece necesario, por tanto, volver a centrar el foco sobre algunas sencillas preguntas: 1) ¿Tuvo España en el último cuarto del siglo XIX y primer tercio del xx una protección alta o baja? Es decir, ¿fue la evolución de la protección en España muy diferente de la ocurrida en otros países? 2) ¿Cuáles fueron las razones y determinantes de la existencia de los niveles de protección españoles y de la mayor o menor protección conseguida por unos sectores a costa de otros? 3) ¿El resultado de estos niveles y de la mayor o menor protección conseguida por unas actividades a costa de otras tuvo consecuencias importantes para el crecimiento económico español o no, y si lo tuvo fue este positivo o negativo?

Este trabajo es parte de un proyecto de investigación más amplio cuya ambición es la de dar una respuesta, aunque sea provisional, a estos interrogantes mediante el estudio en paralelo del caso español e italiano ${ }^{7}$. En estas páginas se realiza un esfuerzo por afrontar el tema de la medición de la protección española en el largo plazo con el objeto de discutir en profundidad la primera pregunta que ha sido planteada con anterioridad. Las dificultades de medición han extendido el método tradicional de evaluar la protección: el estudio de los cambios en las leyes arancelarias a lo largo del tiempo. Este estudio, aunque necesario, no es suficiente, ya que puede introducir fuertes sesgos a la hora de interpretar la tendencia de la protección nominal. Cambios en las leyes arancelarias con aranceles específicos al alza pueden representar, paradójicamente, una reducción de la protección nominal, si se dan las condiciones adecuadas en otras variables. El primer paso, necesario, para el conocimiento y análisis de la protección es inevitablemente el de la medición de la protección nominal ad valorem tanto individual como agregada con el objeto de poder ofrecer

"Ver los trabajos de Costas (1988), Serrano Sanz (1989) y Sabaté (1996).

${ }^{7}$ El proyecto se inició con el caso español, ver Prados-Tena (1994), y se espera pueda concluir en una síntesis de los mismos en el futuro. El estudio sobre el caso italiano aunque se inició con posterioridad ha generado ya alguna publicación, ver Federico-Tena (1998), o Federico-Tena (1999). 
más adelante una perspectiva comparada de la experiencia española, así como el estudio de sus efectos sobre la economía ${ }^{8}$.

La parte central de este artículo la constituye la discusión y análisis de una serie de nuevos indicadores de la protección en España para el período que va de 1870 a 1930 . Estos indicadores no permiten abordar por sí solos el gran tema de la protección y el crecimiento económico pero sí establecer una base sólida que permita profundizar en el conocimiento del nivel, cambios de perfil y carácter del proteccionismo español. Las medidas convencionales ofrecen un nivel de partida alto desde los inicios de la Restauración y el mantenimiento del mismo en términos generales hasta la primera guerra mundial, el nuevo cambio de nivel sólo llegaría en los años veinte con el arancel Cambó. Estos indicadores se presentan junto con otras apreciaciones cualitativas de la literatura en la sección 1, con la intención de discutir abiertamente las contradicciones de nuestro conocimiento actual de la evolución de la protección en España. Las secciones 2 y 3 están dedicadas respectivamente a presentar indicadores alternativos de medición de la protección más adecuados, así como los problemas técnicos y de fiabilidad de su estimación en el caso español. La sección 4 discute los resultados de todos estos ejercicios y confirma que los niveles de protección en España en los inicios de la Restauración eran ya relativamente altos tanto si los comparamos con los inmediatamente posteriores como con los de otros países. Lo que se observa con posterioridad no es un perfil plano si no alcista, aunque cíclico en su evolución. Para terminar se contraponen las conclusiones de este trabajo sobre la tendencia alcista y la continuidad industrial de la política comercial en España con la interpretación del carácter «forzado» o «fortuito» del viraje proteccionista de 1891 , y se exhorta a estudiar la posible ruptura de hecho y no sólo formal entre la instauración de la base quinta con el arancel Figuerola de 1869 y su derogación en 1875.

${ }^{x}$ La primera versión de esta medición se puede ver en Prados-Tena (1994). Para un indicador anual alternativo ver Tirado $(1994,1996)$. La crítica a este último en Tena-Tirado (1996). 


\section{1. ¿QUÉ SABEMOS DE LA EVOLUCIÓN DE LA PROTECCIÓN EN ESPANA?}

El indicador generalmente aceptado como indicativo de la evolución a largo plazo del nivel de protección de una economía es el de la protección nominal y el sistema más usado es el de calcular el porcentaje de los ingresos arancelarios en relación al valor de las importaciones (a partir de ahora NT). Otra opción todavía más discutible, pero también muy difundida por su simplicidad de cálculo, consiste en estimar la evolución de las importaciones como porcentaje del Producto Interior Bruto (OM), asumiendo que los incrementos y decrementos de la demanda afectan en la misma proporción al numerador y denominador de este indicador. Ambos indicadores deberían evolucionar, en terminos muy generales, de forma inversa. Es decir, al incrementar la protección representada por NT se espera una mayor contracción de las importaciones que del PIB y por tanto un decrecimiento de OM. Siendo consciente de la fragilidad de esta relación parece interesante observar la evolución de la política comercial a la luz de una comparación sistemática de ambos indicadores.

El gráfico 1 ofrece una comparación estilizada en términos logarítmicos de ambos indicadores, que en cierta medida confirma la evolución negativa esperada.

Lo más destacado del arancel de 1849 fue la reducción de la lista de prohibiciones y la sustitución de los derechos ad valorem por «específicos», lo que, en un contexto de moderada tendencia al alza de precios, parece haber ayudado a obtener un resultado más librecambista en la polí-

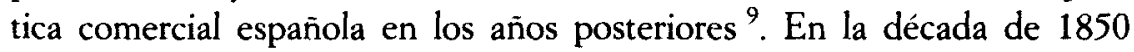
y primera mitad de 1860 ambos indicadores coinciden en ofrecer un período de significativa liberalización de la protección arancelaria. La segunda mitad de los sesenta y la primera de los setenta, aunque el indicador NT sigue en descenso hasta 1872, es más bien un periodo de inestable mantenimiento de las conquistas precedentes. El comienzo de la Restauración con la suspensión de la base quinta de junio de 1875 es recogida por ambos indicadores en el sentido de un incremento de la protección al ofrecer una contracción de las importaciones y un alza de NT respectivamente. Esta tendencia cambia de nuevo a comienzos de la década de los ochenta, con una suavización del NT y una mayor apertura de las importaciones. El

"El indice de precios de las exportaciones inglesas a España elaborado por Prados de la Escosura pasa de 100 en 1850 a 115 en 1864. Prados (1988), cuadro 5-A2, p. 257. Ver también el arancel (1960), p. 21. 


\section{GRÁFICO 1}

Cambios de la protección nominal (NT) y grado de apertura de las importaciones españolas (OM) entre 1850 y 1930

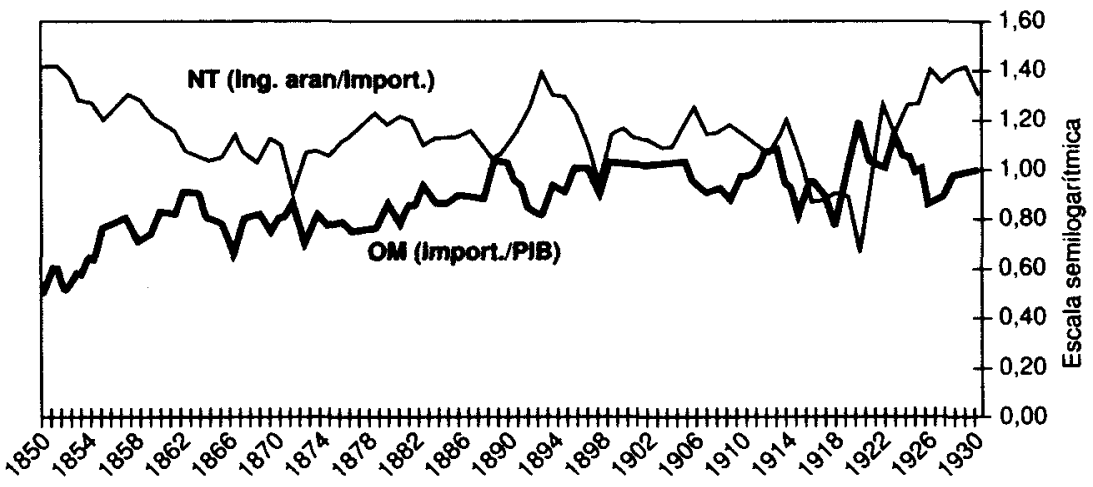

FUENTES: Ingreso arancelario: volúmenes anuales estadísticas del Comercio Exterior, 1850-1930; Importaciones: series corregidas Tena (1989), pp. 341-343; PIB: Prados (1995).

impacto del arancel de 1891 es detectado de forma clara en el comportamiento inverso de ambos índices, aunque durante un período muy breve. En términos generales se puede observar una menor coherencia en el esperado comportamiento inverso de ambos indicadores durante el cambio de siglo, así como una cierta dificultad para evaluar la importancia relativa de las respectivas leyes arancelarias de 1891 y 1906 . El efecto del arancel Cánovas de 1891, aunque sólo dura tres años, es significativo, mientras que el del arancel Salvador de 1906 parece menos relevante. Entre 1923 y 1926, los índices muestran un incremento de la protección nominal acompañada por una contracción significativa de las importaciones. El impacto del arancel Cambó de 1922 sobre ambos índices dura un poco más pero es muy parecido al del arancel Cánovas de 1891.

Otro paso necesario para evitar falsos debates es poner de relieve las diferencias encontradas en las series de ingresos arancelarios y comercio especial más comúnmente usadas por la historiografía.

En el cuadro 1 el denominador de todas las series, salvo en el caso de Tirado, se refiere a las series de importaciones elaboradas por Prados y Tena y recogidas en Tena (1989). Las discrepancias en los resultados 


\section{CUADRO 1}

Indicadores de protección nominal (NT) comúnmente usados (Ingresos arancelarios/Valor importaciones)

\begin{tabular}{|c|c|c|c|c|c|}
\hline & $\begin{array}{c}\text { NT (a) } \\
\text { (Comin) } \\
(\%)\end{array}$ & $\begin{array}{c}\text { NT (b) } \\
\text { (Mitcbell) } \\
(\%)\end{array}$ & $\begin{array}{c}\text { NT (c) } \\
\text { (Tirado) } \\
(\%)\end{array}$ & $\begin{array}{c}N T(d) \\
(\text { Esta. comer. }) \\
(\%)\end{array}$ & $\begin{array}{c}N T(e) \\
(\text { Tena }) \\
(\%)\end{array}$ \\
\hline $1877 \ldots \ldots \ldots \ldots \ldots$ & 16,5 & 16,3 & 14,0 & 12,7 & 12,7 \\
\hline 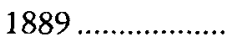 & 14,9 & 14,7 & 11,3 & 10,3 & 11,0 \\
\hline $1897 \ldots \ldots \ldots \ldots \ldots$ & 10,6 & 10,2 & 11,0 & 11,7 & 14,6 \\
\hline $1913 \ldots \ldots \ldots \ldots \ldots$ & 16,3 & 15,9 & 13,4 & 12,0 & 14,9 \\
\hline $1926 \ldots \ldots \ldots \ldots \ldots \ldots$ & 26,3 & 25,4 & n.a & 23,8 & 20,1 \\
\hline
\end{tabular}

Ingresos arancelarios: (a) Comín (1985); (b) Mitchell (1985); (c) Tirado (1996), serie DAOMEPON; (d) Estadísticas del Comercio Exterior; (e) Ingresos arancelarios base de datos de este trabajo. Apéndice, cuadro 1.

Valor importaciones: en todos los casos cifras corregidas de las importaciones (Tena, 1989, pp. 342-343), salvo (c) Tirado (1996), serie DAOMEPON; en (e) se aplica a las importaciones de la muestra el mismo coeficiente corrector implicito en Tena (1989).

del indicador NT tienen su origen, por tanto, en la diversidad de fuentes usadas para obtener los ingresos arancelarios del numerador. En los casos en los que se ha elegido fuentes presupuestarias, como en el de Comín (1985) o Mitchell (1985), se ofrecen resultados con niveles más elevados ${ }^{10}$ que en los casos que se opta por los ingresos arancelarios recogidos de las estadísticas del comercio exterior [estimación (c) de Tirado y la (d) y (e) elaboradas por mí]. Dentro de cada grupo la evolución y los niveles son razonablemente parecidos. Sin embargo, al comparar ambos grupos la percepción del nivel y la evolución puede ser muy distinta. Los dos primeros indicadores del cuadro 1 muestran, en términos generales, niveles más altos que los dos últimos. Esto ocurre en todos los años con la excepción del año 1897. Esto implica que en los dos primeros casos la vuelta al proteccionismo está en mayor medida representada por el año 1877, cuyo nivel de protección no sería superado hasta 1926, mientras en los dos últimos parece más un proceso ascendente desde finales de los años ochenta.

En cualquier caso, sea cual sea el indicador NT usado para medir el nivel de la protección en España lo más relevante del mismo, al compararlo

${ }^{10}$ En principio la renta de aduanas ofrecida de fuentes fiscales está consolidada pero incluye los derechos sobre exportaciones y otros recargos o impuestos no estrictamente arancelarios, como muestra su mayor nivel. 
con la media aritmética de una amplia muestra de países europeos de los que se ha podido obtener información, es que ofrece un nivel más alto y un perfil distinto.

Como se puede observar en el gráfico 2 el NT español muestra en primer lugar un nivel netamente más alto y en segundo lugar un perfil más cercano a una línea cóncava con su parte más deprimida en el centro en contraste con un NT europeo más cercano a un perfil convexo, con su parte más álgida en los años centrales del período.

¿Se puede decir, entonces, que la historia de la evolución del nivel global de la protección en España es sustancialmente distinta a la de la mayoría de los países europeos? Es difícil contestar a esta pregunta sin realizar un trabajo de verificación sobre los sesgos incluidos en el indicador NT tanto en España como en el resto de los países europeos, sin embargo, como primera aproximación se podría decir que si suponemos que el sesgo a la baja del indicador NT es proporcional a su nivel protector sólo podemos esperar un diferencial mayor entre el nivel de la protección nominal en el caso español y europeo. En el caso italiano se ha comprobado la existencia de un perfil moderado de la protección y que la evolución del indicador NT representa de forma aceptable la evolución de la protección en este

\section{GRÁFICO 2}

Nivel y perfil de la protección nominal en España y Europa

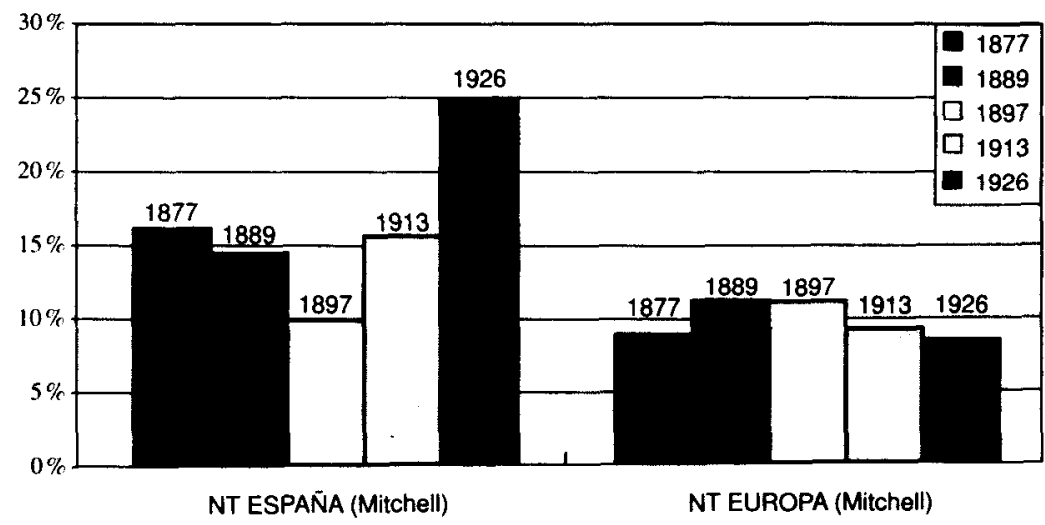

Fuentes: España: cuadro 1; Europa: media aritmética de los NT de Alemania, Rusia, Reino Unido, Francia, Austria-Hungría (Austria en 1926), Italia, España, Bélgica, Holanda, Suecia, Suiza, Dinamarca y Portugal [ver Federico-Tena (1998a, Apéndice, tabla 4]. 
pais ${ }^{11}$. No existen trabajos similares para otros países europeos, y lo que se pretende en las próximas páginas es ofrecer un estudio riguroso sobre el comportamiento del NT en el caso español.

\section{CÓMO MEDIR ADECUADAMENTE LA PROTECCIÓN}

Desde mediados del siglo xIx y hasta la Gran Crisis de los años treinta, las cuotas y otras barreras no arancelarias protectoras del comercio eran prácticamente inexistentes. La política comercial recaía prácticamente en exclusiva sobre los aranceles y, por tanto, la medición del nivel de protección de una economía es comparativamente más fácil que en la actualidad. En principio un arancel produce el efecto de elevar el precio interno del país que lo impone por encima del precio internacional en una cantidad igual a la del mismo arancel ${ }^{12}$. El arancel puede ser expresado como porcentaje del precio internacional (arancel ad valorem), o, como en España y en la generalidad de los países de Europa continental, en relación al peso del producto importado $\mathrm{Ai}$ (arancel específico). En este último caso, por motivos comparativos, es necesario calcularlo como:

$$
\mathrm{Ti}=\mathrm{Ai} / \mathrm{Pi}
$$

donde Ai es el arancel específico y $\mathrm{Pi}$ es el precio mundial ${ }^{13}$.

En la mayoría de trabajos empíricos, los datos sobre aranceles específicos son extraídos de las leyes arancelarias. Esta medida ex ante incluye algún sesgo si los productos implicados han sido importados bajo algún tipo de acuerdo bilateral preferencial, exención o castigo por causa de guerras comerciales. Por este motivo los porcentajes ad valorem es mejor

"Ver Federico y Tena (1998a).

12 En principio, la diferencia entre los dos precios puede ser menor que la cantidad total del arancel (el llamado arancel con agua). Sin embargo este caso no suele crear problemas empíricos en la estimación de la protección con ingresos arancelarios ex-post, ya que este tipo de bienes no es importado.

${ }^{13}$ Anderson (1995) y Feenstra (1995) han argumentado a favor de que la protección debe ser estimada sobre la base de los precios internos con la inclusión de los aranceles -i. e. $T^{*} i=A_{i} /(P i-A i)$ - La diferencia es pequeña, cuando los aranceles no son muy altos, de cualquier forma salirse de la definición tradicional implica no poder comparar los resultados de la estimación con los realizados por la mayoría de los autores para el caso español o con los existentes para otros paises. 
calcularlos en términos ex post como porcentaje de los ingresos arancelarios entre las importaciones reales para cada producto:

$$
\mathrm{PNi}=\left(\mathrm{Qi}^{*} \mathrm{Ai}\right) /\left(\mathrm{Qi}^{*} \mathrm{Pi}_{\mathrm{i}}\right)
$$

El problema real es cómo agregar la protección individual de un producto para llegar a estimar la protección global de una economía completa [Vousden (1990), Greenaway-Milner (1993)]. En principio, los aranceles deben ser ponderados con la estructura de la demanda de importaciones que hubiera existido en condiciones de libre comercio, lo que naturalmente no es un hecho observable. A pesar de algunos avances recientes, la teoría económica no ha suministrado todavía una alternativa exclusiva y totalmente satisfactoria. Es posible ofrecer tres alternativas de ponderación:

a) Prescindir de ponderación (la media arancelaria simple). Como sugirieron la League of Nations (1927) y Liepman (1938):

$$
\mathrm{UNT}=\Sigma \mathrm{T}_{\mathbf{i}} \mathrm{N}
$$

en donde $\mathrm{Ti}=\mathrm{Ai} / \mathrm{Pi}$ y $\mathrm{N}=$ número de productos importados o tasados, según el caso.

b) La estructura real de las importaciones del año en cuestión:

$$
\mathrm{NT}=\Sigma \mathrm{rt}_{\mathrm{i}} * \mathrm{~T}_{\mathrm{i}}
$$

donde $\mathrm{rt}_{\mathrm{i}}$ es la cuota del bien $\mathrm{i}$-th en el total de las importaciones ex post a la imposición del arancel. Por definición puede ser calculado dividiendo el total de los ingresos arancelarios entre el total de las importaciones.

$$
\mathrm{NTt}=\sum_{i=1}^{n}\left(\mathrm{Q}_{\mathrm{it}} * \mathrm{~A}_{\mathrm{it}}\right) / \sum_{i=1}^{n}\left(\mathrm{Q}_{\mathrm{it}} * \mathrm{P}_{\mathrm{it}}\right)
$$

c) La estructura de las importaciones del país en un año anterior a la imposición del arancel, como sugirió McCloskey (1980):

$$
\mathrm{RNTt}=\sum \mathrm{rt}_{\mathrm{i}-1} * \mathrm{~T}_{\mathrm{i}}
$$

en donde $\mathrm{rt}_{\mathrm{i}-1}$ es la cuota del bien $\mathrm{i}$-th en el total de las importaciones ex ante a su imposición. Su cálculo resultaria de la forma siguiente: 


$$
\mathrm{RNTt}=\sum_{i=1}^{n}\left(\mathrm{Qi}_{\mathrm{t}-1} * \mathrm{Ai}_{\mathrm{t}}\right) / \sum_{i=1}^{n}\left(\mathrm{Qi}_{\mathrm{t}-1} * \mathrm{Pi}_{\mathrm{t}-1}\right)
$$

RNT se puede asimilar conceptualmente a un índice de precios Laspeyres, así como NT se puede asimilar a un índice de precios Paasche. Todas estas alternativas introducen algún tipo de sesgo.

El UNT asume que cada producto importado representaría, en libre comercio, la misma cuota de consumo. Es decir, la presunción de una estructura de la demanda no plausible. Cuanto mayor sea la desagregación con que se calcula menor será la dimensión del sesgo respecto a una demanda libre de aranceles. La dimensión del sesgo es inversamente proporcional, como tendencia, al número de productos incluidos en la media [Tumlir-Till (1971)], lo que depende de la distribución de productos incluidos en las estadísticas o recogidos en la base de datos. La tendencia de NT es a sesgar los resultados a la baja, ya que los aranceles reducen la presencia de los productos más protegidos en relación a los menos protegidos. De esta forma se puede decir que la extensión de este sesgo depende de la elasticidad de las importaciones en los grupos de productos con mayor cuota dentro de la demanda de importaciones ${ }^{14}$. Si la elasticidad y la cuota del producto son lo suficientemente altas un incremento de la protección puede ser recogido como una reducción en NT. En este sentido, un país que imponga aranceles prohibitivos a todos los bienes excepto a uno (que lo mantenga libre de aranceles) podría aparecer menos proteccionista que otro que impusiera un arancel uniforme del 5 por 100 sobre todas las importaciones. Por último, RNT es posiblemente la más atractiva de todas las alternativas ofrecidas, si fuera posible encontrar un año cercano con una estructura de las importaciones en situación de libre comercio. Encontrar un año librecambista y cercano en el tiempo en una investigación histórica de largo plazo en el caso español debe ser considerada una tarea muy aproximativa.

Así, es posible concluir que no hay una solución clara y manejable al problema de la medición de la protección. El indicador que mide el «verdadero» nivel de la protección de una economía no puede ser estimado. Se puede reconocer, como en todo problema de números índices, que

${ }^{1+}$ Para que el sesgo sea relevante es condición necesaria, pero no suficiente, que el producto represente una cuota significativa del total de las importaciones, ya que si la elasticidad es muy baja un incremento arancelario importante puede no afectar significativamente a la cantidad importada del mismo. 
no hay una solución ideal y que la mejor solución a la que se puede llegar es un compromiso. Es decir, tener en cuenta los sesgos que introduce cada indicador y tratar de observar si existe una consistencia recíproca en la interpretación conjunta de todos ellos.

Los índicadores propuestos ofrecen además la posibilidad de medir la mayor o menor incidencia de los aranceles, precios o cambios en la composición de la demanda en los cambios en el nivel de protección de un período a otro. La historiografía atribuye a los aranceles el protagonismo de la mayoría de los cambios en la política comercial, pero esta hipótesis no ha sido medida. El nivel de protección puede cambiar también si los aranceles permanecen constantes. Tanto a causa de variaciones en la composición del comercio (como resultado de la misma política comercial o por otras razones) o -en el caso de uso de aranceles específicos- a causa de cambios en el nivel general de precios o de los precios relativos de los diferentes grupos de productos que lo componen.

Así, el cambio en el indicador NT entre un período y otro se puede expresar de la siguiente forma:

$$
\begin{aligned}
{\left[N T_{t}-N T_{t-1}\right]=} & {\left[N T_{t}-R_{N P}\right]+\left[R N P P_{t}-R N T_{t}\right]+} \\
& +\left[R N T_{t}-N T_{t-1}\right]
\end{aligned}
$$

donde NT se define como en (4b), RNT como en (6) y RNP como:

$$
\mathrm{RNPt}=\sum_{i=1}^{n}\left(\mathrm{Qi}_{\mathrm{t}-1} * \mathrm{Ai}_{\mathrm{t}}\right) / \sum_{i=1}^{n}\left(\mathrm{Qi}_{\mathrm{t}-1} * \mathrm{Pi}_{\mathrm{t}}\right)
$$

Cada componente de la derecha de la fórmula (7) mide ceteris paribus cuánto habría cambiado la protección agregada. El primer corchete, o efecto cantidad, mide la responsabilidad de los cambios en la composición de las importaciones (manteniendo los precios y los aranceles constantes) en la variación de NT entre dos períodos consecutivos. El segundo corchete, o efecto precio, mide la responsabilidad de los precios (manteniendo las cantidades y los aranceles constantes). Por último, el tercer corchete, o efecto arancel, mide la responsabilidad de los aranceles (manteniendo constante los precios y las cantidades o lo que es lo mismo la estructura de 
las importaciones del período inicial), es decir, una aproximación al efecto inicialmente previsto de la política arancelaria ${ }^{15}$.

\section{NIVEL, CAMBIOS Y SINGULARIDAD DE LA PROTECCIÓN DURANTE LA RESTAURACIÓN}

Los años elegidos para llevar a cabo esta estimación en el caso español son los de 1877, 1889, 1897, 1913 y 1926 y han sido escogidos atendiendo a dos razones. La primera, minimizar el número de años con la condición de que estos años se situaran antes y después de las leyes arancelarias de 1882, 1891, 1906 y 1922. La segunda, trabajar con años en los que el problema de la sobrevaloración o infravaloración de los valores unitarios de las estadísticas españolas sesgara lo menos posible la estimación de niveles de protección nominal ${ }^{16}$.

${ }^{15}$ Un sistema similar aunque aplicado a series temporales continuas ha sido recientemente usado por Crucini (1994) e Irving (1998), sin embargo su aproximación es menos ambiciosa metodológicamente, ya que sólo toma en consideración el efecto precio y el efecto arancel. En este caso el principal interés reside en captar el efecto desplazamiento de las importaciones.

${ }^{16}$ A los años escogidos se les ha estimado un sesgo agregado de 8,5 por 100 para $1877,4,7$ por 100 para $1889,0,8$ por 100 para $1897,-10,5$ por 100 para 1913 y $-7,7$ por 100 para 1926. En estos años además se ha estimado un sesgo relativamente moderado tanto para productos primarios como para manufacturas. En 1877 un 8,4 por 100 para los productos primarios y un 17,7 para las manufacturas; en 1889 un 6,5 por 100 y un 9,4 por 100 respectivamente, en 1897 un 5,6 por 100 y un $-0,6$ por 100 , en 1913 un $-11,0$ por 100 y un $-4,5$ por 100 y en 1926 un $-7,7$ por 100 y un $-8,5$ por 100 [Fuentes, Prados (1986), Tena, A. (1992)]. El origen de los sesgos en las valoraciones tendría que ver con la sospecha de que los grupos proteccionistas presionaron al alza las valoraciones principalmente de los productos manufacturados con el objeto de hacer aparecer una protección ad valorem nominal más moderada que la real. Esta sobrevaloración se ha detectado sólo para los años anteriores a 1896, en los años que abarca este estudio en las manufacturas sólo parecería relevante para 1877 ya que el sesgo positivo de 1889 no es muy significativo y el resto de los años ofrecen sesgos negativos. Ciertamente, el que el sesgo global de las manufacturas sea negativo no implica necesariamente que dentro de las manufacturas algunos productos (con grupos de presión especialmente influyentes) consiguieran sobrevaloraciones algo mayores que la media de las manufacturas. La negativa a iniciar un trabajo de revaluación de las manufacturas aunque sólo sea de las que arbitrariamente se las suponga de «más alto riesgo de sesgo» se apoya en las siguientes razones: 1) La dificultad de conseguir precios internacionales homogéneos con los de nuestras estadísticas para una variada gama de productos manufacturados [razones expuestas por todos los autores españoles que han trabajado sobre este tema: ver Tortella (1978), Prados $(1982,1986)$ y Tena $(1985,1992)]$. 2) Si los sesgos esperados por la historiografía [excluido Tena $(1985,1992)$ ] son producto de los grupos de presión éstos sólo podrán ser al alza. En ese caso los resultados de este trabajo ofrecería una estimación de la protección nominal de acuerdo a la que el interés 
En los años elegidos, y a partir de los volúmenes oficiales de las estadísticas del comercio exterior, se ha realizado un detallado trabajo de correspondencia entre la clasificación arancelaria por productos española y la Clasificación Uniforme del Comercio Internacional, revisión 2, de las Naciones Unidas (CUCI) para un nivel de cuatro y cinco dígitos ${ }^{17}$. Los volúmenes anuales del comercio exterior antes de 1933 presentan dos denominaciones: «comercio general» y «comercio especial». El primero incluye todas las importaciones para consumo doméstico (directas o a través de puertos francos). El segundo incluye también productos para el consumo doméstico, pero sólo para aquellas categorías admitidas libres de aranceles o bajo un régimen arancelario especial. En principio la suma de ambos coincidiría, de forma bastante ajustada, con el concepto moderno de «comercio especial» y ésta ha sido en principio la población de partida usada ${ }^{18}$. Algunos productos han sido dejados fuera bien por motivos técnicos (como el tipo de unidades en que venían expresados, su falta de correspondencia con números CUCI, etc.) o bien por motivos económicos (como es el caso de algunos productos destinados al consumo monopolístico por parte del Estado y el oro en sus diversas acepciones). Como resultado se ha conseguido una muestra que incluye entre un 80 y un 95 por 100 de la totalidad de los productos de importación registrados por las estadísticas comerciales como comercio de importación total, y prácticamente la totalidad del comercio de importación dedicado al consumo o de «importación especial» (con la exclusión del mencionado grupo de productos excluidos por motivos técnicos). Los resultados de este trabajo se concretan en una matriz de 750 filas correspondientes al mismo número de agrupaciones CUCI de cuatro dígitos. Junto con los números CUCI y el nombre del

de estos grupos pretendia que apareciera, es decir, más moderada de la real. Si se parte del supuesto de que existen razones para pensar que los sesgos de las valoraciones no tienen una tendencia homogénea y que es necesario y posible revalorar individualmente cada producto se puede optar por una vía alternativa, ver Tirado (1994). Una nota crítica a este intento se encuentra en Tena-Tirado (1996).

${ }^{17} \mathrm{La}$ correspondencia ha sido introducida en la base de datos para un nivel de cinco dígitos con la intención de obtener una correspondencia fiable para la agregación de tres dígitos. Este trabajo se ha llevado a cabo mediante el uso sistemático del diccionario de Naciones Unidas (1985) que permite a partir del nombre y características de un producto hacerle corresponder un número de cinco dígitos de la clasificación CUCI (SITC), rev. II, a cada producto incluido en los volúmenes anuales del comercio exterior en los cinco años escogidos.

${ }^{18}$ El «comercio temporal» y la «mercaderías devueltas» han sido excluidas, cuando son identificadas en la estadística como ocurre en los años 1889, 1897 y 1913. En el año 1926 las series de comercio general y especial parecen ya excluir con mayor rigor estos dos conceptos. 
producto se ofrece la siguiente información para cada uno de los cinco años: el número de la clasificación arancelaria, el valor y la cantidad importada, el ingreso arancelario obtenido para cada producto y el arancel específico que por ley le corresponde a cada año ${ }^{19}$. Los precios para cada CUCI (Pji) se han calculado por simple división entre el valor y la cantidad de los mismos (Qji). Asimismo, con el ingreso arancelario y la cantidad importada se han podido calcular los aranceles realmente aplicados para cada número CUCI (Aij).

Para una mejor comprensión e interpretación económica de los niveles arancelarios estimados para los distintos indicadores se ha decidido agrupar los números CUCI, mencionados en el apartado anterior, conforme a la clasificación del GATT (1985/1986). De esta forma se puede llevar acabo un estudio más riguroso por categorías con sentido económico y bien definidas agrupando los productos de la clasificación CUCI dentro de las categorías de: 1) productos primarios; 2) semimanufacturas, y 3) manufacturas industriales, así como de las subsiguientes subdivisiones. Los resultados desagregados según la clasificación GATT de las estimaciones de los indicadores NT, UNT y RNT se ofrecen en el cuadro 1 del Apéndice.

\section{CUADRO 2}

Niveles de protección nominal total en España según diversos indicadores

\begin{tabular}{|c|c|c|c|c|c|c|}
\hline & $N T(1)$ & UNT (2) & RNT (3) & PROM (4) & NT/UNT (5) & NT/RNT (6) \\
\hline 1877 . & 12,7 & 17,7 & n. a. & 15,2 & 0,72 & n. a. \\
\hline $1889 \ldots \ldots \ldots \ldots \ldots$ & 11,0 & 16,7 & 12,0 & 13,2 & 0,66 & 0,91 \\
\hline $1897 \ldots \ldots \ldots \ldots \ldots . . . . . . . . .$. & 14,6 & 26,3 & 17,8 & 19,6 & 0,55 & 0,82 \\
\hline $1913 \ldots \ldots \ldots \ldots \ldots$ & 14,9 & 25,2 & 18,4 & 19,5 & 0,59 & 0,81 \\
\hline $1926 \ldots \ldots \ldots \ldots \ldots . . . . . . . .$. & 20,1 & 34,3 & 33,8 & 29,4 & 0,59 & 0,60 \\
\hline
\end{tabular}

FueNTES: Cuadro 1 Apéndice.

Los niveles globales para estos tres indicadores se resumen en el cuadro 2. Lo primero que cabe destacar es la coincidencia entre los sesgos

${ }^{19}$ En el año 1877 los productos incluidos en las estadísticas no se les hacía corresponder con el número de la clasificación arancelaria como ocurrió con posterioridad. En el año 1926 las estadísticas no ofrecen los ingresos arancelarios por productos, por lo que los ingresos arancelarios han sido estimados a partir de la segunda columna de los aranceles específicos publicados en 1925, es decir los disponibles en 1926 para su aplicación [Consejc de Economía Nacional (1925)] que incluyen todos los aranceles rebajados depués de haber concluido las negociaciones de los tratados comerciales que se llevaron a cabo entre 1921 y 1924. 
esperados en cada indicador y los resultados obtenidos en la estimación de los diversos indicadores. El indicador UNT (nivel arancelario sin ponderar) muestra los valores relativos más altos para el conjunto de los años, como suele ocurrir con este tipo de indicador ${ }^{20}$. El indicador NT (resultante de la ponderación con el valor de las importaciones actuales, ex post) los valores más bajos. El indicador RNT (que pondera el arancel con las cantidades del año anterior a su introducción, ex ante) ofrece en términos generales valores intermedios. Los tres indicadores dan una visión coincidente, en términos generales, aunque los niveles son distintos a grandes rasgos de la evolución y los cambios en los niveles de protección nominal de la economía española.

En la anterior sección se ha mantenido que NT tiende a deprimir en términos generales los verdaderos resultados, y que esto se espera que ocurra en mayor medida conforme el nivel de la protección sea mayor (a igualdad de elasticidades). El cuadro 2 cuantifica las diferencias entre NT y UNT y RNT mostrando cómo la distancia entre estos indicadores se acrecienta claramente a partir de 1897 respecto los años anteriores. El NT de este año muestra la mayor infravaloración de toda la serie en relación con el indicador UNT, y aunque la modera ligeramente ésta se mantiene en los años siguientes. Algo parecido le sucede al indicador RNT para este año y los sucesivos con la particularidad de acentuar este problema para 1926. Estos coeficientes, aunque no recogen los cambios en la evolución temporal de la protección, sí sugieren que la evolución del indicador NT acentúa su infravaloración a partir de la década de los noventa. En este sentido, apoyan la hipótesis, mantenida por la historiografía, de la fuerte acentuación y crecimiento de la protección en España a partir de los primeros años noventa, así como el hecho que el indicador NT sea poco adecuado a la hora de detectar la dimensión de este crecimiento.

El cuadro 2 muestra claramente cómo el perfil del promedio de los tres indicadores tiene algunos rasgos significativamente diversos de los del tradicional indicador NT del gráfico 1 . El resultado para 1877 confirma las impresiones del gráfico 1 al mostrar cómo a pocos años de iniciarse la Restauración existía ya un nivel de protección significativo ${ }^{21}$. Confirma, también, el bajo nivel que mostraba el año 1889 , lo que acentúa el papel moderador del arancel de 1882, la Ley de julio de 1883 y sobre todo

${ }^{20}$ Ver League of Nations (1927), Tumlir y Till (1971).

${ }^{21}$ Compárese también con el bajo nivel obtenido en el caso italiano en 1877 para estos mismos indicadores (NT: 7,3 por 100; UNT: 6,3 por 100). Statistical Apendix, Federico-Tena (1998). 
el efecto de la posterior extensión de los acuerdos comerciales en los años ochenta. La mayor contradicción entre los nuevos indicadores y el gráfico 1 se observa en el año 1897. El indicador NT en este año muestra un incremento moderado aunque significativo en relación al que mostraba el gráfico 1 tanto respecto a 1877 como a 1889 . Además el resto de los indicadores y especialmente UNT muestran un NT especialmente infravalorado para este año. Lo que da coherencia a la contracción brusca, pero temporal, que se observa en el gráfico 1 después de 1891 como consecuencia del freno a las importaciones de un significativo grupo de productos y su paulatina sustitución por otros menos protegidos. Los años siguientes mantienen una pauta parecida aunque con un NT ligeramente menos infravalorado, por lo que en 1913 se mantendría la protección y sólo en 1926 se puede observa un incremento significativo de la misma.

Si se atiende a la media aritmética de los tres índices propuestos (columna 4 del cuadro 2), en 1889 (con un 13,2 por 100) se reduce en más de un 13 por 100 la protección nominal en relación a 1877 (15,2 por $100)$. El incremento más relevante de la protección en los años estudiados se produce entre 1889 (13,2 por 100) y 1897 (19,6 por 100), incremento de un 50 por 100. El dato ofrecido para $1913(19,5$ por 100) muestra, por el contrario, el mantenimiento de la protección para los años siguientes a 1906. En 1926 (29,4 por 100) se incrementa la protección también en un 50 por 100 tanto respecto a 1913 como a los años anteriores a 1906. En síntesis, el cuadro 2 muestra que aunque entre 1877 y 1926 la tendencia de la protección fue claramente creciente estos cambios de nivel se produjeron de forma cíclica con períodos de crecimiento y moderación de la misma. En los años estudiados se puede apreciar un período de moderación entre 1877-1889 y otro de una cierta estabilidad de 1897-1913, en el medio de ambos períodos se sitúa el arancel de 1891, y un cambio notable en el nivel de la protección. Algo parecido sucede con el arancel de 1922 al elevar el nivel de protección entre 1913 y 1926 de forma similar. En este sentido conviene tener en cuenta que 1897 como año representativo del período sería un año bajo mientras 1926, por el contrario, sería un año alto si se atiende a la serie anual del NT (ver gráfico 1).

Este estudio confirma la existencia de un indicador NT sesgado a la hora de mostrar tanto el nivel como la evolución de la protección en España a lo largo del período 1877-1926. En este sentido pudiera parecer útil comparar el indicador de la protección nominal sin ponderar (UNT) en España con la de otro país como Italia, del que disponemos de una estimación similar para estos mismos años. El caso del UNT italiano es espe- 


\section{GRÁFICO 3}

Niveles de protección nominal sin ponderar (UNT)

en España e Italia 1877-1926

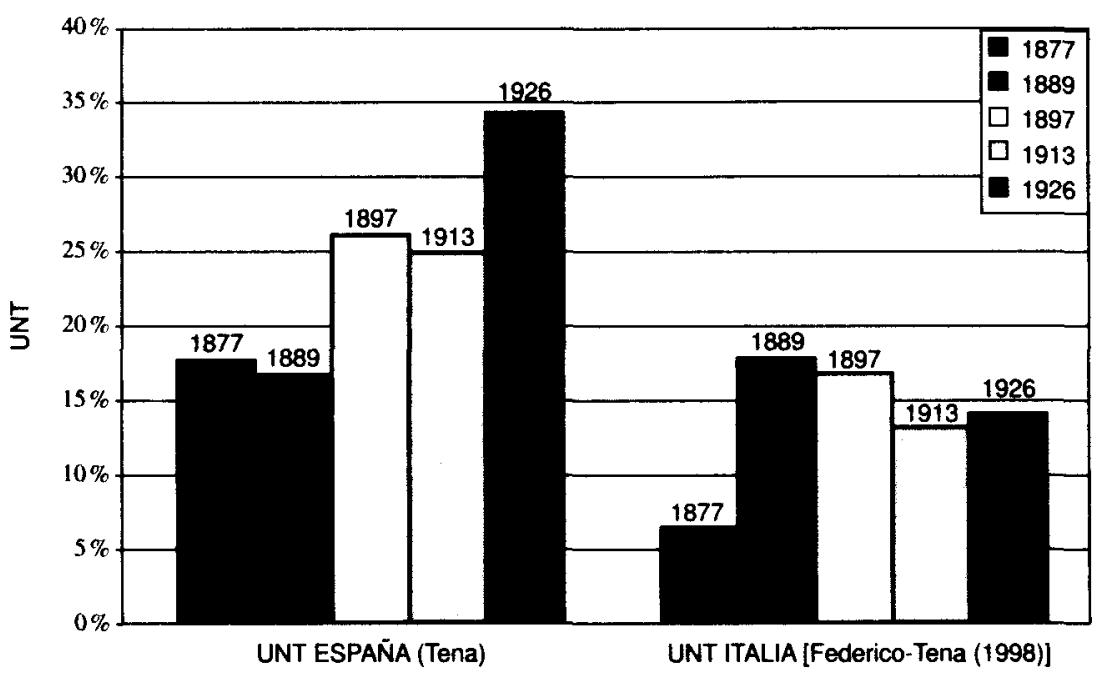

FUENTES: España: cuadro 1 Apéndice; Italia: ver Federico-Tena (1998a, cuadro A1, p. 93.

cialmente significativo ya que su perfil, a diferencia del español, se asemeja tanto asu propio indicador NT como al europeo [ver Federico-Tena (1998a) y gráfico 2]. El nuevo perfil de este gráfico confirma los principales comentarios sobre la singularidad de la evolución de la protección española del gráfico 2. Sin embargo, aporta alguna información adicional interesante. El nivel UNT español es superior en todos los años al UNT italiano salvo para 1889, el año de menor protección española y mayor italiana. El UNT español tiene un punto de partida mucho más alto en 1877 que el italiano y aunque el perfil es más cíclico tiene una tendencia creciente hasta 1926. En España a los años de ligera moderación de 1889 y 1913 le siguen años de fuerte incremento en 1897 y 1922. Por el contrario, Italia con un nivel de partida muy bajo sufre un cambio de nivel en 1889 pero en el período siguiente ya se observa una suavización de la protección, que se mantiene hasta 1926. Aunque el estudio comparado con el caso italiano será merecedor de un próximo trabajo monográfico es importante recordar que el principal incremento arancelario italiano en 1889-1897 tuvo también 
un fuerte componente fiscal (productos con poca elasticidad precio), lo que afectó menos a la infravaloración del NT en relación a los indicadores UNT y RNT ${ }^{22}$. Este contraste, por tanto, reafirma la hipótesis de que el sesgo observado en el indicador NT a partir de 1897 es la consecuencia de la incapacidad del indicador NT para recoger el importante incremento de la protección ocurrida a partir de la década de 1890 en sectores con una alta elasticidad precio.

\section{CARÁCTER Y EXTENSIÓN DE LA PROTECCIÓN EN ESPAN̄A}

Como se vio en la primera sección, la historiografía ha venido discutiendo abundantemente sobre el carácter agrario o industrial de la política arancelaria española. Una primera condición necesaria para saber si hubo una estrategia arancelaria favorecedora de la industria consistiría en poder demostrar que el sector industrial estuvo más protegido que los productos primarios.

\section{CUADRO 3}

Niveles relativos de protección nominal industrial y no industrial en España (IND/NOIND) $\times 100$

\begin{tabular}{|c|c|c|c|c|c|c|c|c|}
\hline & \multicolumn{4}{|c|}{ Industria/No industria } & \multicolumn{4}{|c|}{ Industria/No industria sin coloniales } \\
\hline & $N T$ & UNT & $R N T$ & PROM & $N T$ & UNT & RNT & PROM \\
\hline $1877 \ldots$ & 135 & 125 & - & 129 & 179 & 133 & - & 156 \\
\hline $1889 \ldots \ldots \ldots \ldots$ & 123 & 104 & 70 & 99 & 116 & 106 & 62 & 95 \\
\hline $1897 \ldots \ldots \ldots . . .$. & 95 & 131 & 133 & 120 & 87 & 136 & 117 & 113 \\
\hline $1913 \ldots \ldots \ldots \ldots$ & 91 & 79 & 34 & 68 & 118 & 93 & 49 & 87 \\
\hline $1926 \ldots \ldots \ldots \ldots$ & 112 & 114 & 116 & 114 & 154 & 130 & 139 & 141 \\
\hline
\end{tabular}

FUENTE: Industria: manufactura + semimanufactura. No industria: productos primarios. Ver, Apéndice, cuadro 1; coloniales eliminados los listados en las fuentes del cuadro 7.

El cuadro 3 muestra claramente cómo la protección, tomada en términos relativos, tuvo un perfil industrialista desde el principio de la Restauración en la segunda mitad de los años setenta. Destaca el fuerte carácter industrial del año 1877 y el hecho de que este perfil se suavice o acreciente acompanando la moderación o acentuación de la protección agregada. De

\footnotetext{
${ }^{22}$ Ver Federico-Tena (1998), figura 1, p. 79.
} 
esta forma en coincidencia con la reducción o moderación en el crecimiento del nivel agregado de la protección en los años de 1889 y 1913 se puede observar una pérdida de nitidez del perfil industrial de la protección. Por el contrario en los momentos de mayor auge de la protección agregada como en los años 1897 y 1926 esta nitidez se recupera.

Para una mejor comprensión de los determinantes del nivel de la protección global y de sus cambios es necesario estudiar cuáles son los principales factores que los influencian. El cuadro 1 del Apéndice presenta los resultados obtenidos en NT, UNT y RNT y la media aritmética de los mismos (X) ordenados conforme a la clasificación GATT. A continuación se analizará el perfil que muestra la media de los tres indicadores propuestos $(\mathrm{X})$ con el resultado más convencional ofrecido por el indicador de la protección nominal ponderada (NT). En este sentido se tratara de comprobar en qué medida el indicador NT tiende a reducir la importancia de aquellos productos con aranceles más altos en relación a los indicadores UNT y RNT, y si ésta viene recogida por la diferencia del indicador NT en relación a los otros indicadores. El cuadro 2 del Apéndice explica los cambios en el nivel del indicador NT entre dos períodos a partir de las principales variables que lo determinan: los cambios en la cantidad demandada, los cambios en los precios y los cambios arancelarios. En consecuencia, se van a usar los resultados sectoriales de este cuadro para explicar la influencia del arancel sobre los cambios de la demanda. El cuadro 2 del Apéndice permite corroborar en qué medida la diferencia entre NT y X (la media de los tres indicadores) puede ser achacada a la capacidad del arancel para impedir la entrada a aquellos productos o grupos de productos más fuertemente tasados ${ }^{23}$.

\section{Productos primarios}

Si seguimos el indicador NT los productos primarios (que representan cerca del 50 por 100 del total de las importaciones a lo largo del período) mostrarían un incremento de la protección sólo ligeramente distinta a la que muestra el NT global. Es decir, una protección moderada en 1877 y 1889 y un incremento relevante a partir de 1897 , que se mantiene en

${ }^{23} \mathrm{La}$ información desagregada de todos los productos incluidos en la estimación, junto con su denominación, su protección ad valorem y su importancia ordenada de acuerdo a su CUCI dentro de la clasificación GATT está a disposición de los interesados en papel. Para cualquier otra consulta a la base de datos dirigirse al autor. 
1913 y vuelve a incrementarse ligeramente en 1926 (ver cuadro 1 del Apéndice). En este caso la media de los tres indicadores difiere en mayor medida en lo que atañe al año 1913. La media de los indicadores en el grupo de los productos primarios muestra un incremento importante entre 1877 (13,1 por 100), 1889 (13,5 por 100) y los resultados obtenidos para 1897 (17,5 por 100). En 1913 (con un 23,2 por 100) se obtiene un indicador con una protección sensiblemente más alta (un 33 por 100 de incremento) que en el año precedente del estudio y esta protección continúa creciendo hasta 1926 (con un 27,3 por 100). El principal responsable de este perfil es el de su componente más importante, los alimentos (que representan entre el 20 y el 30 por 100 del total de las importaciones a lo largo del período). Este grupo representa a los productos agrarios alimenticios (es decir a los productos agrarios no utilizados como materias primas en la industria). $\mathrm{El}$ indicador $\mathrm{NT}$ ofrece una protección relativamente alta a los alimentos en 1877 (16,8 por 100) y un sustancial descenso de la misma para 1889 (12,8 por 100). Posteriormente, se observa un fuerte incremento en 1897 (18,6 por 100) y uno mucho más ligero para 1913 (20,6 por 100) para llegar a situar su nivel en 1926 en el 28,1 por 100 . El perfil que muestra la protección a los alimentos, como ocurre en el caso de los productos primarios, se modifica radicalmente si en vez de usar el indicador NT usamos los otros indicadores. Si se usa la media de los tres indicadores, la protección a los alimentos en 1897 (18,9 por 100), aunque ligeramente superior a la de $1889(15,4$ por 100$)$ se mantiene en una magnitud similar a la de 1877 (17,8 por 100). Por el contrario, la media en 1913 (34,2 por 100) supone un incremento de nivel de más del 80 por 100 respecto a 1897 , nivel que se mantiene prácticamente en 1926 (37 por 100). Estos resultados vienen a contradecir la extendida opinión historiográfica de que el arancel de 1906 tuvo por primera vez un carácter industrial respecto a las anteriores leyes arancelarias en sus objetivos protectores y acentúan la necesidad de llevar a cabo un análisis mas detallado y riguroso que permita explicar las razones de esta aparente contradicción.

Las razones que explican, en el grupo de los alimentos, el imperceptible cambio entre el NT de 1897 y el de 1913 se pueden, en parte, aclarar a partir del cuadro 2 del Apéndice. En este cuadro se puede observar cómo los alimentos sufrieron un incremento arancelario del 22 por 100 y que el efecto desplazamiento de la cantidad importada ( $-19,7$ por 100) es el principal responsable de que el NT entre estos dos años sólo recoja una variación del 2 por 100. Es decir el indicador NT en 1913 infravalora los aranceles más altos al ponderarlos con los porcentajes de importación 
más bajos, problema que se evita con los otros indicadores. Este hecho se puede seguir en detalle si se observa la relación entre el incremento de la protección y el freno a la importación de algunos productos alimenticios significativos en 1913, como por ejemplo, la harina de trigo, las conservas alimenticias, la leche y huevos, el pescado, la manteca, los vinos y licores, pero sobre todo artículos alimenticios como el chocolate, la miel, glucosa y dulces, y los considerados coloniales o de renta por las estadísticas como el azúcar, el café, el cacao y las especias ${ }^{24}$. Un apartado especial merece la protección nominal al trigo (que representó a lo largo del primer tercio del siglo $\mathrm{xx}$ alrededor de un 20 por 100 del total de la producción agraria) y que ha monopolizado la visión de la literatura sobre los cambios en la protección agraria total a lo largo del período y especialmente entre 1891 y $1913^{25}$. El régimen arancelario sobre el trigo y sus harinas entre 1892 y 1913 fue muy cambiante y por tanto nuestra elección de fechas anuales para la medición podrían sesgar los resultados sobre la protección relativa del sector. Los datos del GHER (1980) mostrarían que si usamos la media de los derechos arancelarios anuales del trigo de los respectivos períodos en que estuvieron vigentes los aranceles de 1891 y 1906 los aranceles sobre el trigo habrían sido mayores entre 1906 y 1913 que en el período anterior ${ }^{26}$. Por tanto, a pesar de que en los años de este estudio la protección nominal individual del trigo entre

${ }^{24}$ La harina de trigo, cuya protección nominal pasó del 31,4 al 41,7 por 100 entre 1897 y 1913, redujo sus porcentajes sobre el total de las importaciones del 3,57 al 0 por 100; las carnes y conservas alimenticias, entre estos mismos años incrementaron su cuya protección nominal del 17,7 al 33,1 por 100 y redujeron sus porcentajes sobre el total de las importaciones del 0,4 al 0,08 por 100 ; el azúcar de remolacha y caña, cuya protección nominal pasó del 0,44 al 110 por 100 , mientras los porcentajes de importación se reducían del 2,02 al 0,01 por 100; aguas, vino y licores, cuyos porcentajes pasaron del 18,2 al 32,9 por 100 y del 0,37 al 0,2 por 100 respectivamente. Ver Apéndice 2, donde se desagregan los resultados del cuadro 3. Dada su extensión no se adjunta aquí pero está a disposición del lector que lo solicite.

${ }^{25}$ El 20 por 100 de la producción del trigo sobre el total de la producción agraria es una media aproximada de los datos estimados por J. Simpson (1996) para algunos años del periodo 1890-1932. El estudio sobre la protección al trigo del capítulo 4 del libro del GER (1980) sigue siendo probablemente el mejor estudio cuantitativo de que se dispone sobre la evolución de la protección arancelaria y no arancelaria al trigo hasta la primera guerra mundial. Intentos de medición comparativa de la protección (arancelaria y monetaria) del trigo en España en relación a otros países europeos productores a finales del siglo XIX se pueden encontrar en Palafox (1991) y Simpson (1997).

${ }_{26}^{26}$ La media aritmética de los aranceles al trigo entre 1892 y 1905 fue de 6,7 pesetas por hectolitro de trigo y el arancel de 1897 fue de 8,19; por el contrario, la media del período 1906-1913 fue de 7,39 y el arancel al trigo de 1913 de 6,93. Medias calculadas a partir del cuadro 14 en GHER (1980), p. 96. 
1897 y 1913 ofrecen una reducción del 43,7 al 36,4 por 100, estos resultados deberían matizarse si tenemos en cuenta los cambios medios en la protección triguera durante el período 1906-1913.

El hecho de que la media aritmética de los indicadores, a pesar del comportamiento del indicador NT, presente un incremento de la protección en los alimentos entre 1897 y 1913 y que otros indicadores como el de la evolución de la protección arancelaria al trigo confirmen este resultado apoyaría la hipótesis poco ortodoxa de que el arancel de 1906 fue más favorecedor de los intereses agrarios que los aranceles precedentes. Es decir, el arancel de 1906 reforzó la protección arancelaria agraria en contraste con el de 1891 que la elevó sólo ligeramente respecto a los años ochenta pero que en definitiva venía sólo a mantener los altos niveles protectores ya conseguidos en los años setenta. Este incremento de los aranceles de los productos alimenticios está sin duda condicionado por el fuerte incremento que sufrieron el grupo de los llamados coloniales, por razones aparentemente fiscales, a partir de la reforma de Fernández Villaverde de 1899. Lo que se acaba de observar es que hubo un incremento arancelario también en otros productos agrarios alimenticios y que como consecuencia se contrajo sus importaciones, efecto que no recoge el indicador NT. La polémica reside, en parte, en el carácter aparentemente fiscal de la mayor parte de la protección agraria que se acaba de analizar. La caracterización protectora y no fiscal de los aranceles de 1906 y 1911 va a ser motivo de discusión en la siguiente sección.

Las materias primas (como el corcho, cuero, lana, algodón, etc.), como era de esperar, junto con los minerales muestran un perfil protector moderado en todos los años. Los combustibles (ver cuadro 1 del Apéndice) sufren un incremento muy importante en la protección entre 1877 (9,3 por 100) y $1889(44,9$ por 100$)$, que vuelve a elevarse en 1897 (50,6 por 100) y que sólo caerá a niveles cercanos al 33 y 45 por 100 en 1913 y 1926 respectivamente. Los carbones minerales y de cock doblaron su protección entre 1889 ( 5 por 100 para ambos) y $1897(11,4$ y 9,7 por 100 respectivamente) y la volvieron a incrementar más ligeramente en 1913 (13,5 y 11,4$)$ y $1926(14,7$ y 12,5$)$, pero los principales responsables de los altos niveles de 1889 y 1897 fueron los petróleos brutos y rectificados, los aceites rectificados y las vaselinas que en 1889, 1897 y 1913 ofrecen indicadores en muchos casos superiores al 100 por 100 de protección nominal y que sólo moderaron en 1926 (33,6 por 100). La motivación fiscal de estos incrementos arancelarios en los petróleos y aceites y su mayor o menor efecto protector son también motivo de discusión en la siguiente sección. 
Los metales no ferrosos obtienen su protección media más baja en 1889 (con un 10,9 por 100), un incremento importante en 1897 (19 por 100), una ligera reducción en 1913 y una vuelta a niveles cercanos al 25 por 100 en 1926.

\section{Semimanufacturas}

Dentro de las semimanufacturas se encuentran dos importantes grupos de productos de gran significación económica como son la industria del hierro y el acero y gran parte de la industria química. El indicador medio obtenido para el conjunto de las semimanufacturas obtiene su resultado más bajo en 1889 (10,7 por 100). Posteriormente se puede observar un primer incremento importante de cerca del 50 por 100 en $1897(15,3$ por 100 ), el mantenimiento del nivel en 1913 (14,5 por 100) y un fuerte incremento, de casi un 100 por 100 , para el año 1926 (30,9 por 100). El perfil que muestra el indicador NT amortiguaría, los incrementos de la protección que muestra la media de los indicadores para 1897 y 1926. Entre sus componentes el bierro $y$ acero es el que ofrecen un nivel de protección medio y un incremento de la misma más relevante a lo largo de todo el periodo. En 1877 y 1889 se parte de niveles medios del 20 y del 21,6 por 100 respectivamente, y sólo se puede observar un primer incremento relevante de la protección de más del 50 por 100 en 1897 (33,5 por 100). En 1913 (27,1 por 100) se modera ligeramente la protección al hierro y al acero, esta tendencia a la moderación parece romperse radicalmente con un incremento superior al 100 por 100 en el resultado obtenido para 1926 (54,8 por 100). En este caso el indicador NT para el hierro y el acero muestra un perfil algo más alto que la media de los indicadores en los años de 1889 y 1913, y un perfil algo más bajo que la media en 1897 y 1926. En la explicación de los incrementos del indicador NT entre 1889 y 1897, el cuadro 2 del Apéndice resalta de nuevo la contracción de cantidad importada ( $-4,2$ por 100). Es decir, el indicador NT, como en anteriores ocasiones tiende a sesgar a la baja los incrementos en la protección del hierro y el acero, producidos como consecuencia de las Leyes arancelarias de 1891 y sobre todo de la Ley de septiembre de $1896^{27}$.

${ }^{27}$ Desde la Ley General de Ferrocarriles de 1855 y hasta la Ley de septiembre de 1896 la mayoría del material ferroviario disfrutó de un sistema de tarifas arancelarias especiales bastante más reducidas de las consignadas en los aranceles para el resto del hierro y el acero. En 1897 aunque la mayoría del material ferroviario se mantuvo todavía dentro 
Los productos químicos ofrecen una protección mucho más moderada al menos antes de 1926. La media de los indicadores ofrece un perfil moderado, primero en el nivel de partida en 1877 (9,5 por 100) y 1889 (7,6 por 100), y después en los incrementos de 1897 (9,6 por 100) y 1913 (11,5 por 100). El cuadro 2 del Apéndice recoge un fuerte efecto contractivo de las importaciones entre 1913-1926 y algo menor aunque también importante entre 1897-1913. De la misma forma la media de los indicadores (11,5 y 28) se distancia del indicador convencional NT (6,7 y 10) en los respectivos años. Lo que mostraría que tanto en 1926 como en 1913 (aunque en menor medida) hubo una fuerte contracción de las cantidades importadas en los productos químicos más fuertemente protegidos.

\section{Manufacturas industriales}

Las manufacturas no tienen un perfil tan estable como el de las semimanufacturas entre 1877 y 1913 , aunque terminen con un nivel parecido en 1926. Parten con un nivel protector significativamente más alto en 1877 (con un 20 por 100), y sufren un proceso de reducción e incremento de la misma mucho más acentuado en 1889 y 1897, respectivamente $(14,8$ y 25,8 por 100). Posteriormente, a diferencia de la estabilidad que muestran las semimanufacturas, las manufacturas ofrecen una significativa reducción de la protección en 1913 (15,8 por 100). Mientras las semimanufacturas en 1926 ( 31 por 100) ofrecen el indicador con diferencia más alto del período, las manufacturas en 1926 ( 32 por 100), aunque muestran un fuerte incremento respecto a 1913 (15,9 por 100), sólo representan un nivel ligeramente superior al que mantenían en 1897 (25,8 por 100). La diferencia más destacable del indicador NT en relación al resto de los indicadores

del apartado de «comercio especial» los privilegios de este grupo fueron mucho menores y sufrieron una elevación considerable de los aranceles (por ejemplo, en 1896 las barras y carriles del comercio especial tuvieron una protección ad valorem del 13,8 por 100 mientras en 1897 había subido al 28,8 por 100; las «placas de unión» del 12 por 100 pasarón al 53 por 100 , respectivamente). Debido a los cambios introducidos por la Ley de 1896, el año 1897 , en relación al material ferroviario, no es representativo de lo que ocurrió entre 1892 y 1896, pero si de lo que ocurrió en los diez años siguientes hasta 1906. En relación a los cambios arancelarios introducidos por la Ley de septiembre de 1896 respecto al arancel de 1891 y anteriores se puede ver Arana Pérez (1988), p. 8. Las reducciones y franquicias para el material ferroviario estaban recogidas dentro del «comercio general» en el año 1877 . En 1889 y 1897 estos privilegios pasarian a registrarse dentro de un apartado llamado «comercio especial» y el material ferroviario no privilegiado se recoge junto al resto del «comercio general». 
se produce, precisamente, en 1897, de nuevo el año en que la media muestra el incremento de protección más importante del período. El cuadro 2 del Apéndice recoge la importante contracción de la demanda de importaciones que acompaña el fuerte incremento arancelario entre los años 1889 y 1897. Por tanto, tanto la contracción de la demanda como el diferencial NT con la media de los indicadores apuntan a una fuerte contracción de las importaciones como consecuencia del incremento de la protección industrial en 1897. En 1913, por el contrario, el cuadro 2 del Apéndice muestra una reducción arancelaria significativa, amortiguada, en parte, por una bajada de los precios. Lo que da como resultado una bajada de la protección nominal de las manufacturas para ese año. Como resaltaba el cuadro 4, con independencia del indicador elegido, no parece haber duda de que los industriales con el arancel- de 1906 consiguieron una menor protección tanto absoluta como relativa respecto al arancel de 1891 y al de 1926.

Dentro de las manufacturas el grupo 3.1, Bienes de equipo, tienen la particularidad de iniciar el período en 1877 (13,5 por 100) con una protección más moderada que el del conjunto de las manufacturas (20 por 100) y sin embargo terminar el mismo en 1926 (31,4 por 100) con una protección muy similar. Entre estos dos años este nivel se contrae en 1889 (9,3 por 100$)$, se acentúa en 1897 (16,3 por 100) y vuelve a moderarse ligeramente en 1913 (13,9 por 100). En los bienes de equipo se observa sólo un fuerte incremento de la protección a partir de la primera guerra mundial. El apartado Otros bienes de equipo (que incluye piezas y herramientas de hierro, material eléctrico, locomotoras y aparatos de medición) y el apartado Maquinaria especifica de industrias representan el grueso de las importaciones de bienes de equipo y son los principales protagonistas de un cierto efecto de contracción de la demanda en 1913, por lo que mantienen un perfil similar al de su agregado ${ }^{28}$.

El grupo 3.1, Bienes de consumo, muestra una evolución diferente a la de los bienes de equipo, con un nivel de partida mucho más alto en $1877(21,9$ por 100$)$ y un nivel de llegada parecido en 1926 (33 por 100). En 1889 (16,6 por 100) moderan también la protección respecto a 1877 , mientras en 1897 prácticamente la doblan $(30,1$ por 100$)$. El cuadro 2 del Apéndice muestra cómo entre 1889 y 1897 el fuerte incremento arancelario (15,3 por 100) estuvo acompañado de una significativa contracción

${ }^{28}$ Un estudio sobre los niveles de protección e importación de material y maquinaria eléctrica entre 1890 y 1935 se puede ver en Tena (1988). 
de la demanda ( $-8,3$ por 100$)$ que modera el incremento del indicador NT en 1897. El diferencial del indicador NT con el resto de los indicadores recoge también el impacto sobre la demanda de los bienes más fuertemente tasados y por tanto el sesgo a la baja del indicador NT. Este efecto expulsión de la demanda en 1897 se puede observar sobre todo en el caso de uno de sus principales componente, los textiles. Con un incremento arancelario del 16,4 por 100 y reducción de la demanda del 8,4 por 100 es junto con los otros bienes de consumo los más afectados por la infravaloración del indicador NT en el año 1897. La contracción de la demanda parece generalizada entre 1889 y 1897 , haciendo que los textiles pasen de representar el 13,2 al 7,6 por 100 del total de las importaciones españolas entre estos dos años. La media de los indicadores muestra cómo los textiles parten en 1877 (22,9 por 100) con un nivel alto (similar al de su agregado), se moderan algo en 1889 (17,3 por 100), llegan a su máximo de protección en 1897 (con un 32,9 por 100) y posteriormente vuelven a suavizar su protección en 1913 (19,3 por 100), para terminar obteniendo en 1926 $(30,7$ por 100$)$ un resultado parecido aunque ligeramente inferior que en 1897. La confección o vestuario comparten con los tejidos e hilados un punto de partida alto en 1877 (22,6 por 100), pero como le ocurre al acero, la química y a los bienes de equipo obtiene su máximo al final del período en 1926 (40,9 por 100).

En síntesis, los niveles de protección nominal en España a finales de los años setenta, tanto si los comparamos con los posteriores como con los de otros países, eran ya relativamente altos. La responsabilidad del alto nivel de partida es muy amplia pero destaca el protagonismo de las manufacturas de consumo. La década de 1880 ofrece una suavización de la protección producida por los efectos combinados del arancel de 1882, la extensión de acuerdos preferenciales y la Ley de 1883. Con posterioridad, incluso si mantenemos como referencia el alto punto de partida de los años setenta, se puede decir que la protección nominal en España tiene su ruptura arancelaria más importante después del arancel de 1891. La magnitud de este incremento arancelario depende del tipo de indicador utilizado pero en términos relativos no hay duda que el arancel de 1891 representó un incremento significativo de la protección y que este incremento fue protagonizado por las manufacturas industriales. El arancel de 1906, en términos globales, viene a mantener la protección que sólo vuelve a incrementarse de forma significativa en 1926. El estancamiento de la protección en 1906 es el resultado de dos tendencias contrapuestas, por un lado, de la significativa moderación de la protección en las manufacturas 
industriales y, por el otro, del casi igualmente importante incremento de la protección de los productos agrícolas alimenticios. El arancel de 1922 viene a mantener la protección conseguida por los productos agrícolas alimenticios y a recuperar la protección perdida por las manufacturas industriales en relación al arancel de 1906. Es decir, en términos relativos, el arancel de 1891 y el de 1922 otorgaron una mayor protección nominal a la industria y el arancel de 1906, por el contrario, se la otorgó a los productos agrarios (principalmente alimentos). La protección a la industria fue diferente en el arancel de 1891 que en el de 1922. Mientras el primero protegió sobre todo a las industrias tradicionales de los textiles y el hierro el segundo se dirigió especialmente a los sectores manufactureros más acabados (vestuario y otras manufacturas de consumo) junto con los sectores nuevos de la química, bienes de equipo y maquinaria.

La importancia del «efecto cantidad», del «efecto precio» y el efecto «arancel» en los cambios de la protección nominal se han analizado a partir del cuadro 2 del Apéndice. El incremento de los precios jugó un efecto amortiguador importante sólo en el período 1913-1922 y este efecto afectó a todos los sectores menos al de bienes de equipo. Por el contrario, en el período 1897-1913 el efecto precios jugó a favor del incremento de la protección en las manufacturas y semimanufacturas. Los incrementos arancelarios fueron los protagonistas de la tendencia al alza de la protección en todos los períodos y en la mayoría de los sectores, con la natural excepción del período 1877-1889 en que la protección nominal se moderó. La otra excepción la constituye el sector semimanufacturero y sobre todo el manufacturero entre 1897-1913. En este periodo hubo una importante reducción de arancelaria en ambos sectores (sobre todo en los bienes de consumo, maquinaria y hierro y acero) contrarrestada por un protagonismo de los aranceles en el sector primario o más concretamente en el sector alimenticio. El «efecto cantidad» es negativo en todos los períodos y por tanto tiende a moderar el incremento final del indicador NT. Este efecto negativo recoge en parte los cambios en los gustos y precios de los productos, pero también el efecto producido por el arancel al desplazar la demanda hacia los productos menos protejidos. Es decir, es un indicador de la gravedad de los problemas de infravaloración del indicador NT en algunos sectores. Las mayores diferencias entre indicadores se han encontrado en los años en que se producen incrementos significativos de los aranceles que suelen ir acompañados de una contracción de las importaciones. Este hecho se detecta principalmente en el grupo de las manufacturas en 1897 (especialmente los de consumo), en el grupo de los pro- 
ductos primarios en 1913 (especialmente alimentos) y en las semimanufacturas en 1926 (especialmente el grupo de productos químicos). En todos estos casos el resto de los indicadores corroboran una infravaloración del indicador NT como consecuencia del desplazamiento de las importaciones desde los productos más tasados a los menos tasados. Es decir, como era previsible el indicador convencional NT sesga los resultados, en la mayoría de los casos, moderando los incrementos de protección nominal respecto a los teóricamente reales. En este sentido, la disponibilidad de otros indicadores parece permitir ofrecer interpretaciones alternativas más fiables respecto a las que suele presentar la historiografía.

\section{EL COMPONENTE FISCAL Y AGRARIO DEL ARANCEL DE 1906}

Toda la literatura coincide en resaltar cómo después de la pérdida de Cuba y con motivo de la reforma tributaria de Fernández Villaverde en 1899 , dentro del total de las importaciones el grupo de los llamados «coloniales» concentró el interés de los reformadores como fuente de ingre$\operatorname{sos}^{29}$. A continuación se va a analizar el peso y por tanto el efecto de los llamados aranceles «fiscales» en los cambios en la composición de la protección entre el arancel de 1891 y el de 1906. En la anterior sección se ha puesto de manifiesto el significativo incremento de la protección a favor del sector agrario en 1913 tanto en términos absolutos como en relación con las manufacturas. En primer lugar se trata, por tanto, de valorar en qué proporción este hecho se produce como consecuencia del comportamiento arancelario de los llamados productos «coloniales» dentro del sector de productos alimenticios. En segundo lugar, de discutir la complementariedad que en muchos casos se produce entre una razonable intencionalidad fiscal, al incrementar la protección de algunos productos, y su efecto protector sobre la economía.

El cuadro 4 sintetiza el fuerte incremento protector que sufrió el grupo de los llamados coloniales entre 1897 y 1913 y cómo la reducida contracción de sus importaciones permitió una importante mejora de los ingresos arancelarios conseguidos por el grupo. Tanto los ingresos como la tasa arancelaria (medida por NT) se multiplicaron por un factor cercano a 10, lo que significa que el nivel de las importaciones del grupo permaneció prác-

${ }^{29}$ Ver Sabaté (1996) y Comin (1994). 


\section{CUADRO 4}

Cambios en la protección e ingresos arancelarios de los productos «coloniales»

\begin{tabular}{|c|c|c|c|c|}
\hline & $\begin{array}{c}\text { Coloniales } \\
\text { NT } \\
(\%)\end{array}$ & $\begin{array}{l}\text { Total } \\
\text { NT } \\
(\%)\end{array}$ & $\begin{array}{c}\text { Ingresos aran. } \\
\text { coloniales } \\
\text { (mill. ptas.) }\end{array}$ & $\begin{array}{c}\text { Ingresos aran. } \\
\text { Total } \\
\text { (mill. ptas.) }\end{array}$ \\
\hline $1897 \ldots \ldots \ldots \ldots \ldots$ & 7,4 & 14,6 & 3,0 & 105,4 \\
\hline $1913 \ldots \ldots \ldots \ldots \ldots$ & 67,9 & 14,9 & 30,3 & 189,6 \\
\hline
\end{tabular}

FuENTE: Estadísticas del Comercio Exterior, grupo coloniales, compuesto en 1897 por azúcar extranjera, glucosa, caramelo líquido, azúcar colonial, cacao extranjero, cacao colonial, cacao molido, café extranjero, café colonial, café molido, canela Ceilán, canelas otras, clavo, nuez moscada con y sin, pimienta, té, vainilla.

ticamente constante. Este incremento de los ingresos supuso, a su vez, prácticamente un tercio del incremento de los ingresos totales ${ }^{30}$.

En la sección anterior comprobamos que el uso de otros indicadores distintos del NT resaltaban la existencia de un fuerte giro de la política protectora entre el arancel Cánovas y el arancel Salvador desde las manufacturas a los productos agrarios alimenticios. A continuación se van a realizar dos sencillos ejercicios para tratar de evaluar el peso que tuvo esta manifiesta intención «fiscal» sobre los cambios en la protección que tuvieron lugar entre 1897 y 1913. Para ello se va a realizar un sencillo «contrafactual» con la intención de valorar qué hubiera ocurrido con la protección relativa entre los principales sectores implicados si la protección de este grupo de productos, que la literatura caracteriza como de fiscales, no hubiera variado entre 1897 y 1913, o en el caso de que simplemente no hubiera existido este grupo de productos dentro del sector alimenticio.

Los resultados del cuadro 5 muestran claramente el importante efecto del llamado grupo de productos «coloniales» a la hora de explicar el brusco giro de la política arancelaria en un principio claramente favorecedora de los productos industriales a una protección relativa fuertemente favorecedora de los productos agrarios. El apartado $\mathrm{A}$, muestra lo que ocurrió realmente (con la inclusión de los coloniales) y por tanto ofrece los mismos resultados discutidos en la sección anterior. El apartado B) explica qué

30) Sabaté (1996), p. 87, cuantifica la contribución del incremento del ingreso por coloniales entre $1895-99$ y $1900-1904$ en un 7 por 100 sobre el total de los ingresos ordinarios del Estado. En este caso la contribución al crecimiento es del 32 por 100, si bien es cierto que a diferencia de Sabaté se realiza entre 1897, dos antes de la reforma Villaverde, y 1913, año en que también influyó el recargo de $10 \mathrm{ptas} / 100 \mathrm{~kg}$. que se impuso (por la Ley de 24 de diciembre de 1912) a los llamados coloniales (excluido el azúcar). 


\section{CUADRO 5}

Impacto de los llamados «productos coloniales» en los indicadores relativos de protección (media aritmética entre los indicadores NT, UNT y RNT)

\begin{tabular}{|c|c|c|c|c|}
\hline & \multicolumn{4}{|c|}{ A) Con coloniales } \\
\hline & $\begin{array}{c}\text { Alim. (1) } \\
(\%)\end{array}$ & $\begin{array}{c}\text { Manuf. (2) } \\
(\%)\end{array}$ & $\begin{array}{c}\text { Total (3) } \\
(\%)\end{array}$ & $(1) /(2)(4)$ \\
\hline $1897 \ldots \ldots \ldots \ldots \ldots$ & 18,9 & 25,8 & 19,6 & 0,73 \\
\hline \multirow[t]{3}{*}{$1913 \ldots \ldots \ldots \ldots \ldots$} & 34,2 & 15,9 & 19,5 & 2,2 \\
\hline & \multicolumn{4}{|c|}{ B) Colon. $13=$ Colon 97} \\
\hline & $\begin{array}{c}\text { Alim. (5) } \\
(\%)\end{array}$ & $\begin{array}{c}\text { Manuf. (6) } \\
(\%)\end{array}$ & $\begin{array}{c}\text { Total (7) } \\
(\%)\end{array}$ & $(5) /(6)(8)$ \\
\hline $1897 \ldots \ldots \ldots \ldots \ldots \ldots$ & 18,9 & 25,8 & 19,6 & 0,73 \\
\hline \multirow[t]{3}{*}{$1913 \ldots \ldots \ldots \ldots \ldots$} & 19,5 & 15,9 & 16,5 & 1,2 \\
\hline & \multicolumn{4}{|c|}{ C) Sin coloniales } \\
\hline & $\underset{(\%)}{\text { Alim. (9) }}$ & $\begin{array}{c}\text { Manuf. (10) } \\
(\%)\end{array}$ & $\begin{array}{c}\text { Total (11) } \\
(\%)\end{array}$ & $(9) /(10)(12)$ \\
\hline $1897 \ldots \ldots \ldots \ldots \ldots$ & 21,8 & 25,8 & 20,3 & 0,85 \\
\hline $1913 \ldots \ldots \ldots \ldots \ldots$ & 21,8 & 15,9 & 16,3 & 1,38 \\
\hline
\end{tabular}

FUENTEs: Simulación obtenida a partir de la misma base de datos con que se elaboró el cuadro 1 del Apéndice. Se ha considerado productos coloniales a los mismos que figuran en tal categoría en las estadísticas oficiales de 1897: azúcar extranjera, glucosa, caramelo liquido, azúcar colonial, cacao extranjero, cacao colonial, cacao molido, café extranjero, café colonial, café molido, canela Ceilán, canelas otras, clavo, nuez moscada con y sin, pimienta, té, vainilla.

hubiera ocurrido en 1913 si los aranceles sobre los «coloniales» se hubieran mantenido como en 1897, suponiendo también la demanda constante. El apartado C) simula lo que hubiera ocurrido con los indicadores relativos de la protección en ausencia del grupo de productos coloniales en ambos años. Los dos últimos apartados reducen de forma neta el nivel de protección medio conseguido por los alimentos tanto si mantenemos la protección sobre los coloniales como si prescindimos de los mismos. La protección sobre los alimentos sería en este caso muy similar en 1897 a la que se conseguiría en 1913. Si asumimos que los productos coloniales 
son todos «productos fiscales» sin efecto protector y que por tanto deben ser excluidos del cálculo de la protección las conclusiones serían las siguientes. En primer lugar se puede observar que el grupo de los coloniales aporta casi tres puntos al alza al nivel total de la protección en 1913 mientras que en 1897, por el contrario, lo reduciría ligeramente. En segundo lugar, la exclusión o mantenimiento de la presión arancelaria sobre los coloniales permite observar cómo el importante grupo de los alimentos no coloniales como mínimo mantuvo la protección entre estos dos períodos. Si a esto se añade la pérdida significativa de protección del sector manufacturero, no hay ninguna duda de que el arancel de 1906 mejoró la posición relativa del sector agrario en relación al manufacturero. El hecho más significativo que pone de manifiesto este ejercicio es que, en cualquier caso, tanto si aceptamos la asunción extrema de excluir a los coloniales como si no, la reducción de la protección de las manufacturas aparece como el factor más relevante a la hora de explicar la ganancia relativa de protección del sector agrario entre 1897 y 1913.

Es necesario reconocer que aunque la literatura se ha concentrado en los productos coloniales la caracterización de los llamados «artículos de renta» es mucho más amplia. El problema reside en que cualquier producto de consumo generalizado y con una demanda más bien inelástica es susceptible de ser considerado un producto fiscal (como le ocurrió al trigo en 1906$)^{31}$. La dificultad de separar un efecto fiscal de uno protector

${ }^{31}$ Tradicionalmente algunos estudiosos opinan que es necesario excluir los llamados «artículos de renta» de los cálculos de protección nominal, ya que su imposición arancelaria se haria por motivos fiscales y no por motivos de protección. El que el legislador declare su intención fiscal al imponer un arancel no excluye que este arancel tenga consecuencias protectoras. Algunos de los llamados «artículos de renta» eran producidos en el interior del país o contaban con sustitutos directos. En otros casos, es decir cuando los productos no son producidos en el interior y no cuentan con sustitutos directos aparentes, las consecuencias de un arancel sólo afectarian directamente al bienestar de los consumidores e indirectamente a la asignación de recursos. A los primeros mediante la reducción de la renta y al segundo incentivando la producción de sustitutos indirectos (por ejemplo, un alto arancel sobre el café, el cacao o la canela puede favorecer la producción de achicoria o de especies de origen mediterráneo) o de sus insumos. Es decir, no se puede justificar la exclusión de ningún grupo de productos por motivos fiscales exclusivamente, y en cualquier caso la exclusión ha de ser individual y fundamentada económicamente. En el caso de España, como expone Serrano Sanz (1991), pp. 115-116, ni siquiera la literatura oficial ofrece una definición doctrinal: - «Como tampoco había una definición doctrinal clara de lo que había de entenderse por "artículo de renta", los contornos del grupo se van difuminando progresivamente; y el número de productos incluidos no cesa de aumentar. De un lado, se agregan ciertas mercancías de las que no hay producción interior y sí un comercio creciente, como el petróleo o algunos productos químicos. De otro, bienes que proporcionan una recaudación crecida, aunque se produzcan también aquí, como el trigo. Lo cierto es 
hace muy difícil reunir a un grupo de productos o «artículos de renta» cuyos aranceles no tengan efecto protector. La inclusión o no de estos últimos en las estimaciones de la protección es parte de un debate recien$\mathrm{te}^{32}$. La producción dentro del país de sustitutos muy directos de algunos productos coloniales o la consideración como tales de algunos productos con algún grado de manufacturación y por tanto potencialmente producibles, aconseja realizar otro ejercicio que permita clarificar al menos los términos en que debe plantearse el debate ${ }^{33}$. Un segundo ejercicio consistiría en intentar elegir con criterios económicos y no administrativos el grupo de productos que más se acercaría al impreciso concepto de productos fiscales sin efecto protector. Para ello en el cuadro 6 se presenta el impacto que hubiera tenido sobre la protección relativa de los alimentos la exclusión de este grupo de un número significativo de productos alimenticios o combustibles no elaborados que comparten la característica de no contar con producción o sustitutos muy directos dentro del país. Los artículos de la estadística excluidos han sido los siguientes: café en grano sin tostar, cacao en grano, té y té imitación hierba, pimienta, canela, tabaco hoja para fábricas, tabaco en rama, aceite de coco y los petróleos brutos y sus aceites.

Este último ejercicio confirmaría a grandes rasgos las reflexiones que se derivaron al excluir el grupo de los productos coloniales del cuadro 6. Naturalmente, el hecho de mantener algunos productos agrarios con producción interior cuya protección fue incrementada también con intenciones

que al discutirse la siguiente reforma de bases arancelaria en 1906, se identifican hasta setenta partidas como de renta, frente a las 22 de 1869."

${ }^{32}$ Muy recientemente en Nye (1991) se argumenta la necesidad de incluir para medir la protección británica (en los primeros tres cuartos del siglo $\mathrm{xIX}$ ) los llamados aranceles de renta sobre vinos y licores, para recoger el efecto protectivo sobre sus sustitutos en el consumo popular como la cerveza [ver también su reciente polémica a este respecto con Douglas Irving (marzo 1993)].

${ }^{33}$ En el caso español existen ejemplos muy claros de productos coloniales que tienen sustitutos directos como es el caso de la azúcar de remolacha y el azúcar de caña. El efecto protector sobre el sector del azúcar de remolacha que supuso el incremento del warancel renta» sobre la caña de azúcar a partir de la Ley de diciembre de 1899 se pueden ver en Jiménez Blanco (1986). Hay otros casos menos claros y menos estudiados pero donde el incremento de precios por los «aranceles renta» en productos coloniales como la canela, pimienta y el clavo pudo tener efectos protectores sobre especias de producción nacional como azafrán, coinino, orégano, pimiento molido. Algo parecido se podría decir también del café, cacao o té, que como el vino con la cerveza en el caso inglés también pudieron disponer de sustitutos en el consumo popular (achicoria...). Cualquier elección parece arbitraria y en este caso se ha optado por la opción tradicional de excluir a un grupo significativo de productos que no disponían de producción nacional del mismo producto o de un sustituto muy directo. Ver texto. 


\section{CUADRO 6}

Impacto de la exclusión de un grupo de «productos fiscales» en los indicadores relativos de protección (media aritmética entre los indicadores NT, UNT y RNT)

\begin{tabular}{|c|c|c|c|c|c|c|c|c|}
\hline & \multicolumn{4}{|c|}{ A) Con prod. fiscales } & \multicolumn{4}{|c|}{ B) Sin «prod. fiscales» } \\
\hline & $\begin{array}{c}\text { Alim } \\
(1) \\
(\%)\end{array}$ & $\begin{array}{c}\text { Manuf. } \\
(2) \\
(\%)\end{array}$ & $\begin{array}{c}\text { Total } \\
(3) \\
(\%)\end{array}$ & $\begin{array}{c}(1) /(2) \\
(4)\end{array}$ & $\begin{array}{l}\text { Alim. } \\
\text { (1) } \\
(\%)\end{array}$ & $\begin{array}{c}\text { Manuf. } \\
(2) \\
(\%)\end{array}$ & $\begin{array}{c}\text { Total } \\
(3) \\
(\%)\end{array}$ & $\begin{array}{c}(1) /(2) \\
(4)\end{array}$ \\
\hline $1897 \ldots \ldots \ldots$ & 18,9 & 25,8 & 19,6 & 0,73 & 21,1 & 25,8 & 19,1 & 0,82 \\
\hline $1913 \ldots \ldots \ldots$ & 34,2 & 15,9 & 19,5 & 2,2 & 30,7 & 15,9 & 17,5 & 1,9 \\
\hline
\end{tabular}

FuENTES: Simulación estimada a partir de la misma base de datos con que se elaboró el cuadro 3.

fiscales en 1913 da como resultado un incremento mucho más importante de la protección del sector agrario que en el cuadro 5. Es decir, entre 1897 y 1913, independientemente de las motivaciones aducidas, se puede constatar un incremento significativo de la protección nominal no sólo de todos los productos agrarios de consumo sino también del amplio grupo que contaba con producción en el interior del país. Este segundo ejercicio, por tanto, no sólo confirmaría y reforzaría la hipótesis de la existencia de un cambio a favor de la agricultura en relación a su protección relativa con la industria en 1913, como se confirmaba en el cuadro 5, sino también de su protección absoluta en relación al período anterior.

\section{CONCLUSIONES}

Las evidencias aportadas en las secciones precedentes contradicen en parte algunas opiniones muy extendidas en la historiografía reciente sobre la evolución y el perfil de la protección arancelaria en España durante el período de la Restauración. Este estudio ofrece, por primera vez, una visión global de la evolución de la protección nominal tanto agregada como intersectorial mediante el uso de un grupo de nuevos indicadores. En un próximo trabajo se presentarán indicadores de protección efectiva cuyos resultados, se puede adelantar, coinciden a grandes rasgos con los presentados en este trabajo. Aunque las mediciones han sido hechas sólo para algunos años, una proyección de los sesgos estimados sobre una serie temporal completa permite aventurar algunas hipotesis razonables: 1) Que aun- 
que la protección después de 1875 sigue un perfil cíclico ascendente, no todos los ciclos son iguales y el más pronunciado se produce después de una cierta moderación en los años ochenta con la llegada del arancel Cánovas de 1891. 2) El arancel de 1906 parece mantener o suavizar muy ligeramente la protección para posteriormente continuar su tendencia al alza desde los inicios de los años veinte con el arancel de 1922, aunque este segundo ciclo tiene un perfil ligeramente más suave que el observado para los años noventa del siglo XIX. 3) En términos generales se puede decir que la protección durante todo el período de la Restauración aparece con un sesgo netamente industrial y que esto se puede detectar desde sus inicios en 1877. 4) La moderación o acentuación de este sesgo industrial parece estar en conexión con la suavización o incremento del nivel general de la protección agregada. La industria sale ganando con los aranceles que acentúan más claramente la protección y la agricultura con los que la relajan o moderan. 5) Aunque durante todo el período de la Restauración la protección se pueda caracterizar de industrial, contrariamente a lo que mantienen los estudios más recientes, el arancel Cánovas de 1891 aparece con un sesgo industrial muy superior al del arancel Salvador de 1906.

Estos hechos, razonablemente probados, permiten poner en discusión algunas hipóteis muy extendidas en la historigrafía española. La continuidad del carácter industrial resaltada por este trabajo choca frontalmente con la interpretación del carácter «forzado» o «fortuito» del viraje proteccionista de 1891, tan popular últimamente entre algunos historiadores económicos españoles ${ }^{34}$. Lo cual no entra en contradicción con la importancia de los esfuerzos llevados a cabo por parte de la Administración en defensa de los intereses exportadores ni con el hecho de que el fracaso de las negociaciones tuviera su papel a la hora de reforzar las posiciones de los sectores industriales proteccionistas. La negociación del tratado comercial con Francia, sin duda influyó en el diseño del arancel de 1891 pero fueron otras las fuerzas que determinaron su configuración final al acentuar su carácter industrial y mantenerlo durante un período tan dilatado ${ }^{35}$.

${ }^{34}$ Ver Serrano Sanz (1987), Sabaté (1996), Nadal-Sudrià (1993), Comín (1993), J. Pan Montojo (1994), Tirado (1996a), etc.

${ }^{35}$ Otras muchas leyes arancelarias del periodo en Europa fueron diseñados en el fragor de una guerra comercial con Francia en este mismo periodo y a pesar de partir de presupuestos negociadores muy parecidos al español es posible observar un perfil y un carácter netamente distintos. Existe una amplia literatura internacional que pone de manifiesto, cómo en los siglos XIX y XX este tipo de proceso, tanto de liberalización como de acentuación del proteccionismo, se ha desarrollado en el marco de complejas negociaciones comerciales bilaterales o multilaterales. En todos los casos, el objetivo de mantener negociaciones entre 
En el arancel Salvador de 1906 los hechos muestran que mientras se reajustaba y moderaba ligeramente la protección industrial, los productos agrarios recuperaron un cierto protagonismo tanto por incrementar el número y el nivel de los productos protegidos como por el significativo incremento de los llamados productos coloniales. Esto no niega, sino que confirma, que en ambos casos, el incremento de la protección a los productos primarios viniera arropada por la mayor preocupación y protagonismo de las intenciones recaudatorias del Estado desde la reforma Villaverde de 1899. Que la preocupación fiscal influyera no significa que sea el factor determinante a la hora de caracterizar el proteccionismo español desde los inicios del siglo xx. Prueba de ello la da el arancel Cambó de 1922 en donde destaca el significativo incremento arancelario generalizado de todos los sectores y el renovado protagonismo del sector industrial. Existió, por tanto, una cierta continuidad en la política protectora de la Restauración antes y después del arancel Cánovas de 1891. Es decir, contrariamente a lo que se ha venido manteniendo recientemente, toda la evidencia aportada tiende a conformar la idea de que la protección española tuvo en gran medida un sesgo industrial desde los inicios del período restaurador. Este hecho abre el interrogante sobre el mayor o menor grado de continuidad del nivel protector de la economía española entre la Restauración y el sexenio liberal, volviendo a plantear si existió una ruptura de hecho y no sólo intencional entre la instauración de la base quinta con el arancel Figuerola y su derogación en 1875.

En consecuencia parece verse reforzada en mayor medida la hipótesis tradicional sugerida por Vicens Vives según la cual el arancel Cánovas de 1891 reforzó los intereses de los sectores industriales tradicionales del textil y del acero (del textil en 1891 y del acero especialmente después de 1896) y que fueron éstos quienes lideraron la coalición con los representantes agrarios ${ }^{36}$. No hay duda de que en la elaboración de una estructura aran-

gobiernos es el de reducir, lo más posible, el daño causado por el incremento de barreras a los productos de exportación nacionales en los mercados extranjeros.

${ }^{36}$ «La crisis de 1886 dio oportunidad a los proteccionistas catalanes para sumar a su causa a los metalúrgicos vascos y a los cerealistas castellanos. Así se ganó la batalla de los aranceles de 1891 y se establecieron las bases de la articulación proteccionista de la economía española. La pérdida de las colonias en 1898 acabó de remachar el clavo, y preparó la adopción de los aranceles de 1906», Vicens Vives (1987), p. 638. Aún más explícito es Raimon Carr (1990): «Los proteccionistas que habian fracasado con el tratado francés de 1882, tuvieron éxito en 1892 cuando el tratado con Alemania fue rechazado por el Senado. Con la Liga Nacional de Productores, Cataluña había conseguido crear finalmente, tras cuarenta años de propaganda, una organización "nacional" en favor de la 
celaria influyen factores no intencionales como las relaciones comerciales, las necesidades fiscales, las coyunturas económicas, etc., y que todos ellos contribuyen a reforzar o debilitar a los diversos grupos de interés en conflicto. El mecanismo político que permitió este resultado ya sea mediante la presión o la falta de presión de los principales grupos interesados es sin duda uno de los debates más interesantes que abre esta investigación.

\section{BIBLIOGRAFIA}

ANDERSON, J. (1994): «The theory of protection», in D. GREENAWAY, L. A. WINTERS (eds.), Surveys in International trade, Oxford, Basil Blackwell, pp. 107-138.

ARANA PÉREZ, I. (1988): «El empresariado siderometalúrgico vasco y la Ley de septiembre de 1896», Actas del II Congreso Mundial Vasco, Congreso de Historia de Euskal Herria, t. V, San Sebastián.

Arancel (1960): «El arancel en la economía española», Información Comercial Española, núm. 322, junio 1960.

CARRERAS, A. (1984): «La producción industrial española, 1842-1981: la construcción de un índice anual», Revista de Historia Económica, año II, núm. 1, pp. 127-57.

CARR, R. (1990, 1966): España, 1808-1975, Barcelona, Ariel.

Comin, F. (1985): Fuentes cuantitativas para el estudio del sector puiblico en España, 1801-1980, Madrid, IEF.

- (1993): «Política fiscal y proteccionismo en España», La economia política del proteccionismo, V Congreso de la Asociación de Historia Económica, San Sebastián, 29 de septiembre al 1 de octubre de 1993.

Consejo de Economia Nacional (1925): Aranceles de aduanas para la peninsula e islas Baleares y repertorio para su aplicación.

CosTAS, A. (1988): Apogeo del liberalismo en «La Gloriosa». La reforma económica del Sexsenio liberal (1868-1874), Madrid, Siglo XXI de España.

CrucinI, J. M. (1994): «Sources of Variation in Real Tariff Rates: the United States, 1900-1940», American Economic Review, vol. 84, núm. 3, pp. 732-743.

Federico, G.; TENA, A. (1998): «Was Italy a proteccionist country?», European Review of Economic History, vol. 2, núm. 1, pp. 73.97.

- (1999): «Did trade policy foster italian industrialization. Evidences from the efective protection rates 1870-1930», Research in Economic History, volume 19, pp. 111-138.

Flores de Lemus, A. (1928): Diario de Sesiones, 20 de enero de 1928, «Sobre la política arancelaria española: un debate en la Asamblea Nacional de la Dictadura», reproducidas en Hacienda Pública Española, núm. 42-43, 1976.

protección que incluía a los intereses del acero vasco y a los intereses trigueros castellanos.» La aparente contradicción de una España mayoritariamente agraria en donde la industria protagoniza las demandas de protección es resuelta en términos «olsonianos» en P. Fraile (1991). 
Fralle, P. (1991): Industrialización y Grupos de Presión. La economia politica de la protección en España, 1900-1950, Madrid, Alianza.

FEenstra, R. C. (1995): «Estimating the effects of trade policy», in G. Grossman, K. RoGofF (eds.), Handbook of International Economics, 3, Amsterdam, Elsevier North-Holland, pp. 1553-1592.

GHER (GRuPO dE Estudios DE Historia RuRAL) (1980): Los precios del trigo y la cebada en España, 1891-1907, Madrid, Banco de España.

Irving, D. A. (1993): «Free Trade and Protection in Nineteenth-Century Britain and France Revisited: A Comment on Nye», Journal of Economic History, vol. 53, núm. 1, pp. 146-158.

JIMÉNEZ BLANCO, J. I. (1986): «La remolacha y los problemas de la industria azucarera en España, 1800-1914», en Garrabou, Barciela y Jimenez Blanco (eds.), Historia agraria de la España contemporánea, 3, El fin de la agricultura tradicional (1900-1960), Barcelona, pp. 280-316.

League of Nations (1927): Tariff Levels Indices, Geneva, pp. 1-38.

MCCloskey, D. (1980): «Magnanimous Albion: Free Trade and British National Income, 1841-1881», Exploration in Economic History, vol. 17, pp. 303-320.

Mrtchell, B. R. (1985, 1975): Eumpean Historical Statistics, 1750-1980, Londres, MacMillan.

NACiONES UNIDAs (1985): «Índices de los productos de la Clasificación Uniforme del Comercio Internacional», Informes Estadísticos, serie M, núm. 38/Rev., vol. I, pp. 1-559, y vol. II, pp. 1-495.

NADAL, J.; SUDRlà (1993): «La controversia en torno al atraso económico español en la segunda mitad del siglo XIX (1860-1913)», Revista de Historia Industrial, núm. 3, pp. 199-227.

NyE, J. V. (1991): «The Myth of Free-Trade, Britain and Fortress France: Tariffs and Trade in the Nineteenth Century», Joumal of Economic History, núm. 51, pp. 23-46.

PAN Montojo, J. (1994): «El vino y la política comercial en la Restauración», en Agricultura y Sociedad, núm. 72, pp. 167-203.

Prados de la Escosura, L. (1982): Comercio exterior y crecimiento económico en España, 1826-1913: tendencias a largo plazo, Madrid, Banco de España.

- (1988): De imperio a nación. Crecimiento y atraso económico en España (1780-1930), Madrid, Alianza.

Prados de la Escosura, L.; Tena, A. (1994): «Proteccionism in Spain, 1869-1930, Eleventh International Economic Congress, Milán, 12-17 de septiembre de 1994, pp. 63-76.

Prados de la Escosura, L. (1995): «Spain's Gross Domestic Product, 1850-1993: Quantitative Conjectures», Working Paper 95-05 y 95-06, Economics Series, 01.

Palafox, J. (1991): Atraso económico y democracia. La Segunda República y la economía española, 1892-1936, Barcelona, Crítica.

SABATÉ (1996): El proteccionismo legitimado. Política arancelaria española a comienzos de siglo, Zaragoza, Civitas.

SerRANo SANZ, J. M. (1987): El viraje proteccionista en la Restauración. La política comercial española, 1875-1895, Madrid, Siglo XXI.

- (1989): «El proteccionismo y el desarrollo económico en la Restauración. Reflexiones para un debate», Revista de Historia Económica, año VII, núm. 1. 
- (1991): «La renta de aduanas en España (1849-1935)», Hacienda Publica Española, núm. 1, pp. 107-119.

SimpSON, J. (1997): «Did Tariffs stifle Spanish agriculture before 1936», European Review of Economic History, vol. 1, part 1, april, pp. 65-87.

- (1996): Spanish Agriculture: The Long Siesta 1765-1965, Cambridge University Press.

TENA, A. (1988): «Importación, niveles de protección y producción de material eléctrico en España, 1890-1935», Revista de Historia Económica, núm. 2, pp. 341-371.

- (1989): «Comercio exterior», en Carreras, A. (ed.): Estadísticas Históricas de España, siglos XIX-XX, Madrid, Fundación Banco Exterior, pp. 331-361.

- (1991): «Protección y competitividad en España e Italia, 1890-1960», en PRADOS, L., y ZamaGni, V., El desarrollo económico en la Europa del Sur: España e Italia en perspectiva bistórica, Madrid, Alianza, pp. 321-355.

- (1992): «Las estadísticas históricas del comercio internacional: fiabilidad y comparabilidad», Banco de España, Estudios de Historia Económica, núm. 24.

- (1995): «The Spanish Foreign Sector, 1885-1985», en Martin-Aceña y Simpson (eds.), The Economic Development of Spain since 1870, Edward Elgar, pp. $402-418$.

TENA-TiRADO (1996): «Protección arancelaria en la Restauración. Un debate Antonio Tena-Daniel Tirado», Revista de Economía Aplicada, núm. 11 (vol. IV), pp. 135-150.

TIRADO, D. (1994): «La protección arancelaria en la Restauración. Nuevos indicadores», Revista de Economia Aplicada, núm. 6 (vol. II), pp. 183-203.

- (1996): La Protección arancelaria en la Restauración: impactos de corto y largo plazo. España, 1870-1913, Tesis Doctoral, Departament d'Historia i Institucions Económiques, Universitat de Barcelona, marzo 1996.

Tortella, G. (1985a), en García Delgado (ed.), La España de la Restauración. Politica, economía, legislación y cultura, Madrid, Siglo XXI.

- (1994): El desarrollo de la España contemporánea. Historia económica de los siglos XIX y XX, Madrid, Alianza.

TumliR, J.; Tul, L. (1971): «Tariff Averaging in International Comparisons», en Grubel, H. G.; Johnson (eds.), Efective Tariff Protection, Ginebra.

Vicens Vives, J. (1987, 1959): Manual de Historia Económica de España, Barcelona, Vicens Vives. 


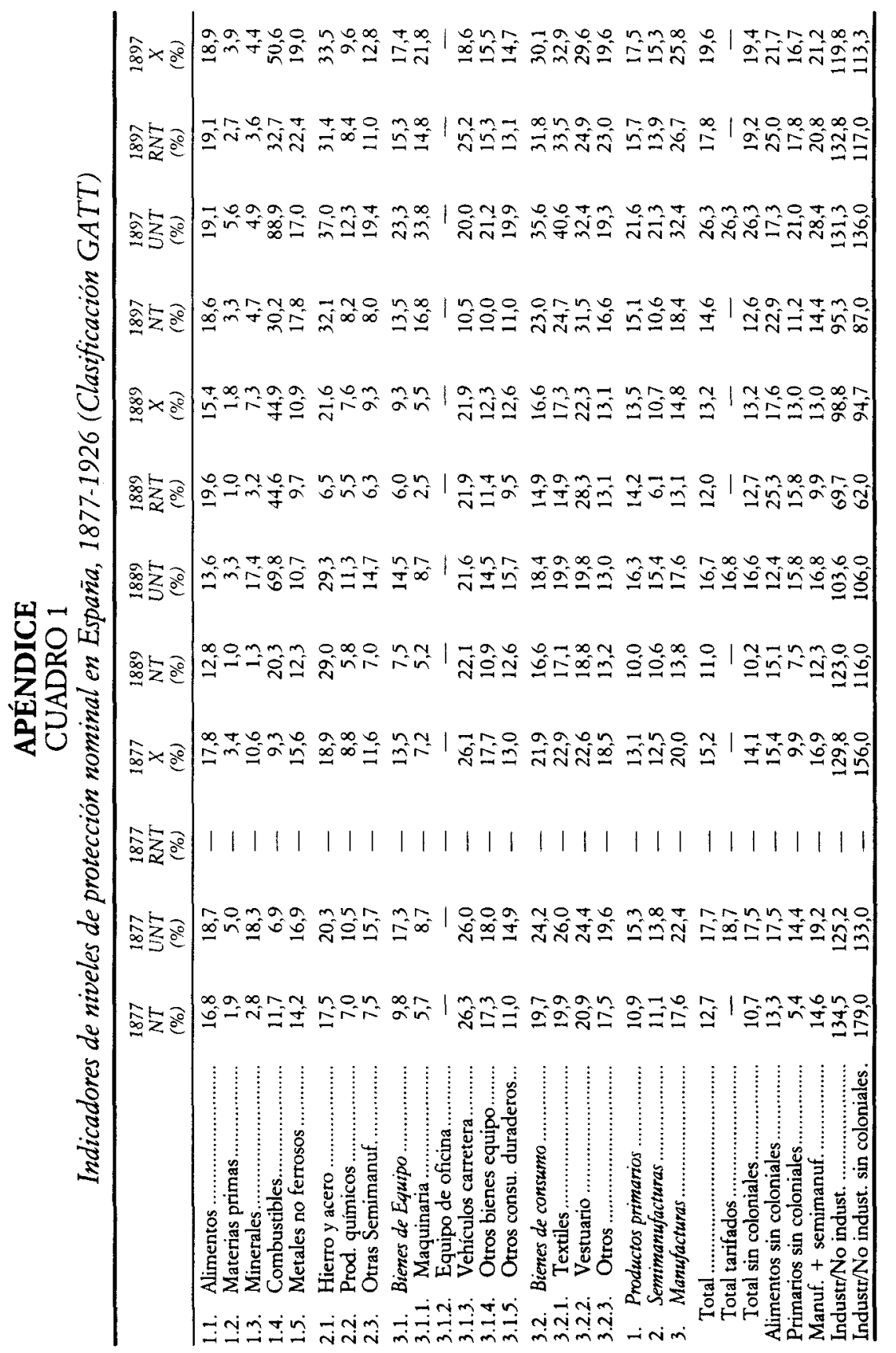




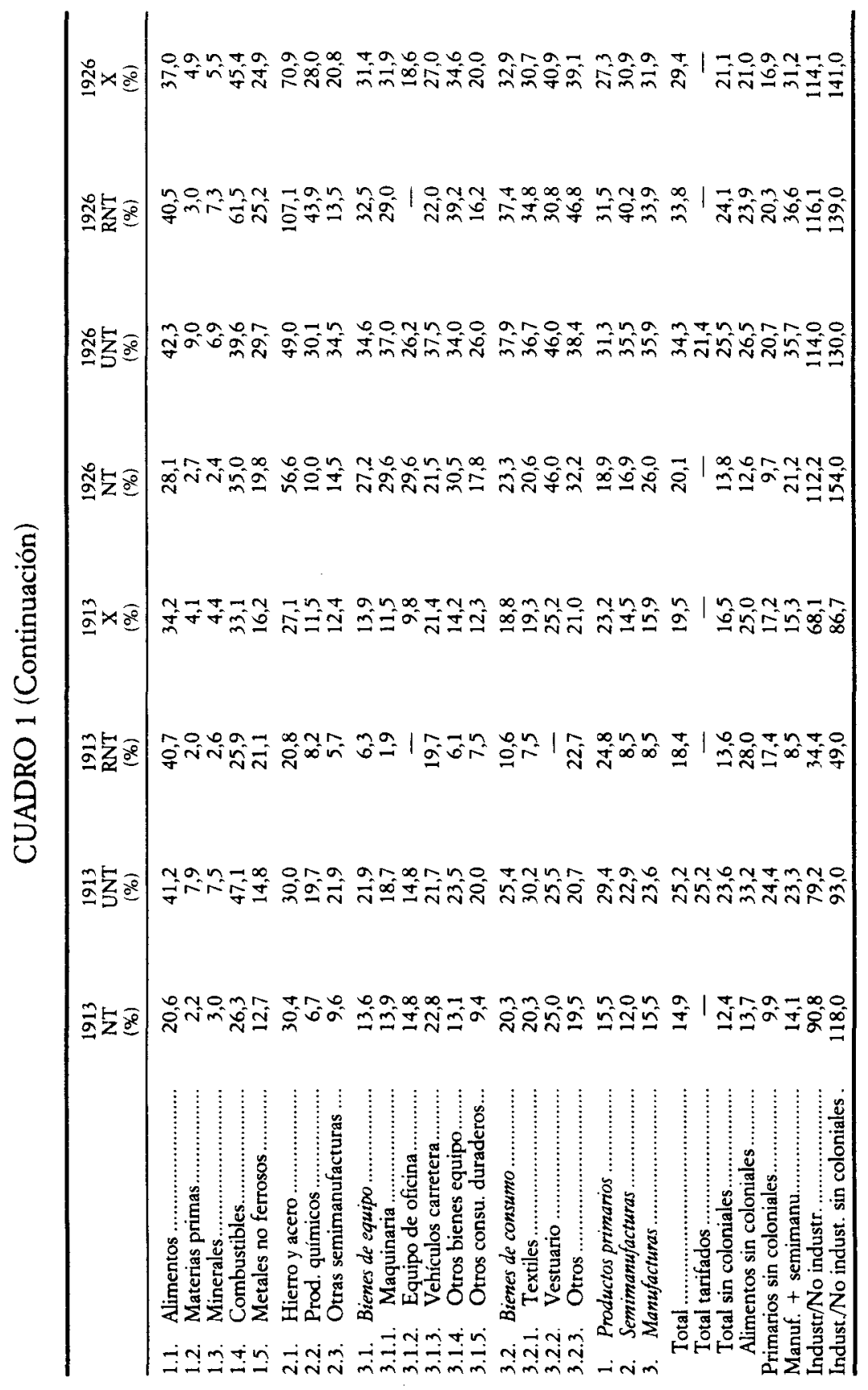




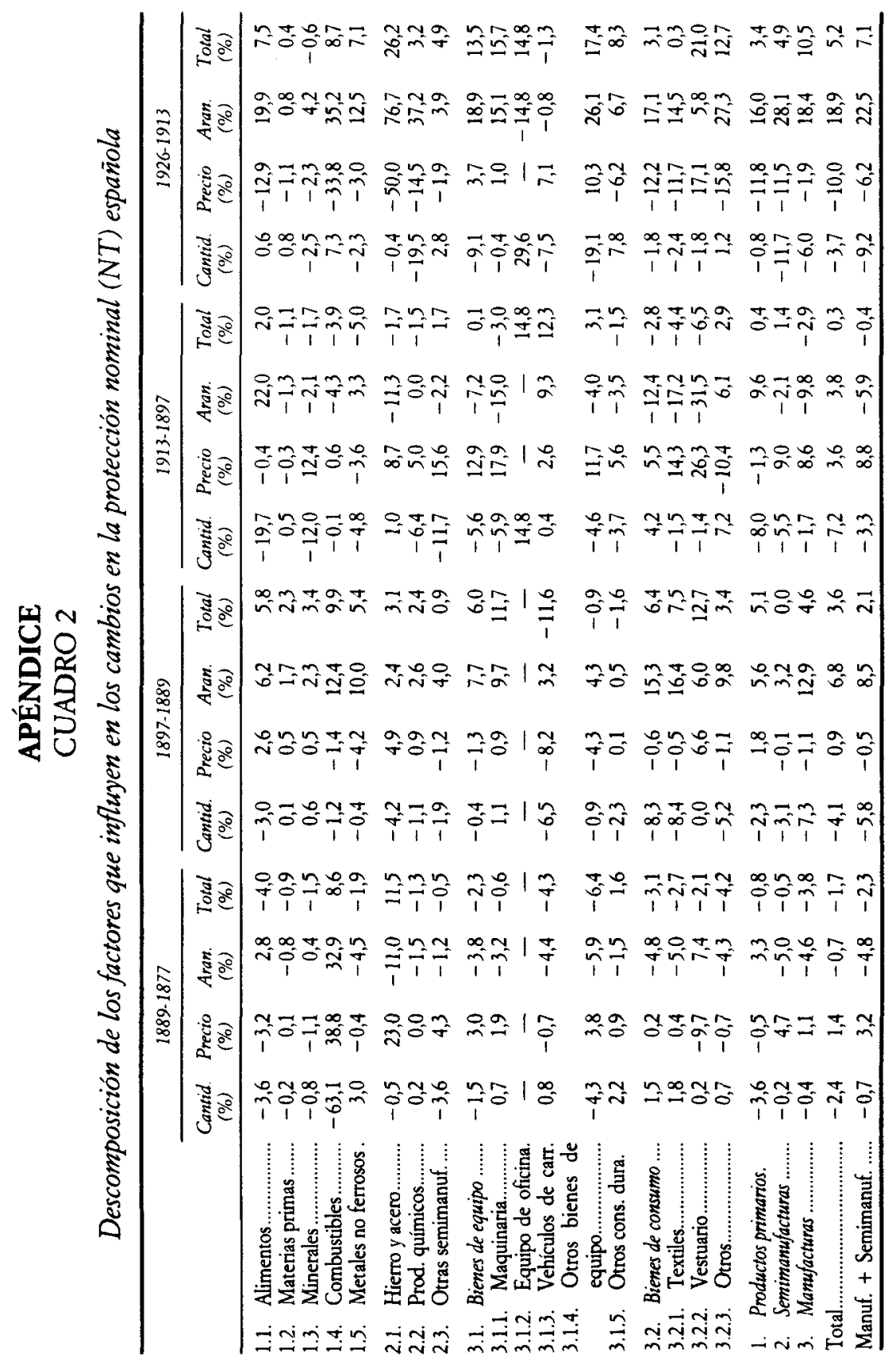


